IUCM-95046

\title{
Scaling theory of the Kondo screening cloud
}

\author{
Erik S. Sørensen \\ Department of Physics, Indiana University, Bloomington, IN 47405 \\ Ian Affleck \\ Department of Physics and Canadian Institute for Advanced Research \\ University of British Columbia, Vancouver, BC, V6T 1Z1, Canada
}

(September 9, 2018)

\begin{abstract}
A scaling form for the local susceptibility, derived from renormalization group arguments, is proposed. The scale over which the uniform part of this scaling form varies can be viewed as a definition of the Kondo "screening cloud" $\sim \xi_{K}$. The proposed scaling form interpolates between Ruderman-KittelKasuya-Yosida (RKKY) results in the high temperature limit, $T \gg T_{K}$, and Fermi liquid results in the low temperature, long-distance limit, $T \ll T_{K}$, $r \gg \xi_{K}$. The predicted form of the Knight shift is longer range at low temperatures where the screening cloud has formed, than at high temperatures where it has not. Using weak and strong coupling perturbation theory combined with large scale density matrix renormalization group (DMRG) results we study the validity of the finite size version of the scaling form at $T=0$. We explicitly extract a length scale proportional to the Kondo length scale, $\xi_{K}$. The numerical results are in good agreement with the proposed scaling
\end{abstract}


form and confirm the existence of the Kondo screening cloud.

75.20.Hr, 75.10.Lp, 75.40.Mg

Typeset using REVTEX 


\section{INTRODUCTION}

The Kondo effect is probably one of the most well-studied phenomena in condensed matter physics. The highly successful theoretical approaches include Wilson's numerical renormalization group (NRG) method国, simpler and more physical renormalization group (RG) approaches of Anderson et al. 1 and Nozières 3 and exact Bethe ansatz results 1 . Thus it is perhaps surprising that a fundamental aspect of this problem remains mired in controversy.

From the RG viewpoint, the Kondo effect is associated with very large distance scales, $\xi_{K} \approx a e^{1 / \rho J}$, where $a$ is the lattice spacing, $\rho$ the electronic density of states at the Fermi surface and $J$ the Kondo coupling. This scale is essentially $\xi_{K} \approx v_{F} / T_{K} \approx a E_{F} / T_{K}$, where $v_{F}$ is the Fermi velocity, $E_{F}$ the Fermi energy and $T_{K}$, the Kondo temperature, is the energy scale associated with the Kondo effect. Since Kondo temperatures are normally of order 10 's of degrees, this scale is normally thousands of lattice spacings (ie. microns). A heuristic description of the $R G$ results on the Kondo problem says that a cloud of electrons of this order of magnitude surrounds the impurity spin, forming a singlet with it. The remaining low-energy electronic excitations outside the screening cloud do not "feel" the impurity spin. Rather the screened complex acts like a potential-scatterer for these electrons, with a unitary limit phase shift of $\pi / 2$ right at the Fermi energy. The largeness of this Kondo length scale, $\xi_{K}$, in experimental systems in which the Kondo effect is apparently observed is rather disconcerting. Even a very dilute system with 1 part per million of impurities has a typical inter-impurity separation of about 100 lattice spacings, much smaller than $\xi_{K}$. Thus each impurity has many other impurities inside its screening cloud and it is surprising that

the single-impurity Kondo effect is observed at all. Nonetheless, the impurity resistivity, susceptibility, etc. are observed to be linear in impurity concentration and these quantities seem to fit theoretical expectations.

Comparatively little of the theoretical work on the Kondo effect has focussed on spatial correlations. These seem to be difficult to obtain using Wilson's method and impossible from the Bethe ansatz. Perturbative calculations have been performed $\mathrm{B}$ as have calcula- 
tions using the "Nagoaka equations" a type of mean field theoryl. Renormalization group approaches have been developed by Chen et al.10 and Ganl 8 . However, Chen et al.10 only consider short-range correlations, with $r \ll \xi_{K}$ and do not address the issue of the size of the screening cloud. We incorporate the perturbative results into our discussion of the renormalization group and scaling, but come to rather different conclusions than Gan $\mathrm{B}$ about the scaling variables and the size of the screening cloud. Related theoretical work has addressed the screening cloud in the Anderson model using various approximate methods 1 世 3 . Some of the previous results have been reviewed in Ref. 14, and 15 .

Experiments which have attempted to look for this large screening cloud have obtained mixed result16.17. In particular, the NMR experiments of Boyce and Slichtert were interpreted to indicate the absence of this cloud. This has led to some theoretical discussion about the circumstances under which this cloud can be observed and even to some doubts about its existence.

The purpose of this paper is to examine in more detail, the behavior of the Knight shift (ie. the electronic spin polarization by an applied field) in the vicinity of a magnetic impurity. In the next section we make a scaling hypothesis about this quantity based on standard $R G$ arguments and assuming the existence of a large screening cloud. We point out the rather unintuitive result that the Knight shift is actually longer range at low temperatures, where the screening cloud has formed, than at high temperatures where it has not. We argue that the NMR experiments are not necessarily in contradiction with our scaling form. The basic problem with the experiments, according to this view, is that they only probed very short distances, $<3 a$, whereas $\xi_{K}$ is presumably thousands of times larger than $a$.

We then test our scaling hypothesis numerically. This is done using a one-dimensional tight-binding model. We don't expect that the reduced dimensionality is important since the Kondo problem is intrinsically one-dimensional anyway. Taking a spherically symmetric dispersion relation and a $\delta$-function Kondo interaction, we may decompose the electronic degrees of freedom into spherical harmonics. Only the s-wave interacts, and this corresponds to a one-dimensional problem. The numerical method we use basically restricts us to $T=0$ 
and a finite length, $L \leq 40-50$. As will be seen, this finite length plays essentially the role of an inverse temperature in our scaling arguments. Both the reduced dimensionality and the finite length may be directly relevant to more recent experiments 18 which have attempted to find the screening cloud using small samples with lengths of order $\xi_{K}$ or smaller. Recent theoretical work has also addressed these issues 19 .

In Section II we present the scaling form for the local susceptibility. Renormalization group arguments are given bridging a high temperature RKKY form with the low temperature long distance form in a single scaling expression. Sections III and IV briefly discuss the form of the Hamiltonian we use in our numerical work and some details of the numerical method. Weak and strong coupling perturbation results are presented in section VI and VII along with DMRG results. Finally in Section VIII the cross over region is studied and the scaling form is tested.

\section{RENORMALIZATION GROUP ARGUMENTS}

We consider the standard Kondo model:

$$
H=\sum_{\mathbf{k}} \epsilon_{k} \psi_{\mathbf{k}}^{\dagger \alpha} \psi_{\mathbf{k} \alpha}+J \mathbf{S}_{\mathrm{imp}} \cdot \sum_{\mathbf{k}, \mathbf{k}^{\prime}} \psi_{\mathbf{k}}^{\dagger \alpha} \frac{\boldsymbol{\sigma}_{\alpha}^{\beta}}{2} \psi_{\mathbf{k}^{\prime} \beta} .
$$

Here $\psi_{\mathbf{k} \alpha}$ is the annihilation operator for conduction electrons of momentum $\mathbf{k}$, spin $\alpha$. In the following we suppress spin indices which are implicitly summed over. $\mathbf{S}_{\mathrm{imp}}$ is the impurity spin operator of magnitude $s=1 / 2$. The $\sigma^{a}$ 's are Pauli matrices, and we set $\hbar=1$. The total spin operator is:

$$
\mathbf{S}_{\mathrm{tot}}=\mathbf{S}_{\mathrm{imp}}+\sum_{\mathbf{k}} \psi_{\mathbf{k}}^{\dagger} \frac{\boldsymbol{\sigma}}{2} \psi_{\mathbf{k}}
$$

(We assume, for simplicity, equal g-factors for the impurity spin and conduction electrons.) The Knight shift is proportional to the local susceptibility:

$$
\chi(r, T) \equiv(1 / T)<\psi^{\dagger}(\mathbf{r}) \frac{\sigma^{z}}{2} \psi(\mathbf{r}) S_{\text {tot }}^{z}>
$$


This consists of a bulk part, the usual Pauli susceptibility, $\rho / 2$, where $\rho$ is the density of states per spin, together with a local part arising from the impurity. The total change in the susceptibility due to the impurity, usually called the impurity susceptibility, $\chi_{\mathrm{imp}}$, is:

$$
\chi_{\mathrm{imp}}=\int d^{3} r[\chi(r)-\rho / 2]+(1 / T)<S_{\mathrm{imp}}^{z} S_{\mathrm{tot}}^{z}>
$$

Lowest order perturbation theory gives the RKKY result, which becomes, at $r k_{F} \gg 1$ :

$$
\chi(r, T)=\frac{\rho}{2}+\frac{\lambda}{16 r^{2} v_{F} \sinh \frac{2 \pi r T}{v_{F}}} \cos 2 k_{F} r .
$$

Here $\lambda$ is the dimensionless coupling constant,

$$
\lambda \equiv \rho J
$$

In the limit, $r \ll v_{F} / T$, (but still $r \gg 1 / k_{F}$ ) this gives the well-known RKKY expression:

$$
\chi-\frac{\rho}{2} \rightarrow \frac{\lambda}{32 \pi r^{3} T} \cos 2 k_{F} r
$$

A crucial feature of the Kondo problem is that, for antiferromagnetic coupling, the Kondo coupling increases under renormalization as the energy scale is reduced. The lowest order renormalization group equation:

$$
d \lambda / d \ln \Lambda=-\lambda^{2}
$$

is obtained. Here $\Lambda$ is the momentum space cut-off, or effective band-width. This gives the effective coupling at momentum scale $\Lambda$ :

$$
\lambda_{\mathrm{eff}}(\Lambda)=\frac{\lambda}{1-\lambda \ln \left(\Lambda_{0} / \Lambda\right)}
$$

Here $\Lambda_{0} \approx 1 / a$ is the bare cut-off and $\lambda$ is the bare coupling (defined at that scale). The Kondo length scale is defined from the momentum scale at which the effective Kondo coupling constant diverges:

$$
\xi_{K}=v_{F} / T_{K} \approx \Lambda_{0}^{-1} e^{1 / \lambda}
$$


A finite temperature acts as an infrared cut-off on perturbation theory so that Eq. (2.9) with $\Lambda$ replaced by $T / v_{F}$ can be used to define a temperature-dependent effective coupling.

Eq. (2.5) is only valid at high temperatures and weak Kondo coupling. As the temperature is lowered, the effective Kondo coupling increases so higher order terms become important. The corrections of $O\left(\lambda^{2}\right)$ has been calculated. From Eq. (2.2), we see that $\chi(r, T)$ is a sum of two terms:

$$
\begin{aligned}
\chi(r)= & \int_{0}^{\beta} d \tau<\psi^{\dagger}(\mathbf{r}, 0) \frac{\sigma^{z}}{2} \psi(\mathbf{r}) S_{\mathrm{imp}}^{z}(\tau)> \\
& +\int_{0}^{\beta} d \tau<\psi^{\dagger}(\mathbf{r}) \frac{\sigma^{z}}{2} \psi(\mathbf{r}, 0) \int d^{3} r^{\prime} \psi^{\dagger}\left(\mathbf{r}^{\prime}, \tau\right) \frac{\sigma^{z}}{2} \psi\left(\mathbf{r}^{\prime}, \tau\right)> \\
\equiv & \chi_{d e}(r)+\chi_{e}(r) .
\end{aligned}
$$

We have adopted the notation of Ref. ( 8 ). Note that the sum of these correlation functions is independent of $\tau$ since the total spin is conserved, so that the $\tau$-integral simply gives a factor of $\beta$. However, the individual correlation functions depend non-trivially on $\tau$. We could equally well add the equal time correlation functions and multiply by $\beta$, but we choose the above representation because both terms have been evaluated explicitly in the literature, in a convenient form. The RKKY term of $O(\lambda)$ in eq. (2.5), comes entirely from $\chi_{d e}$. In the asymptotic region, $r k_{F} \gg 1, E_{F} / T \gg 1$, with $r \ll v_{F} / T$, including the correction of $O\left(\lambda^{2}\right)$ :

$$
\chi(r)-\frac{\rho}{2}=\frac{\cos 2 k_{F} r}{32 \pi r^{3} T}\left\{\lambda+\lambda^{2}\left[\ln \left(k_{F} r\right)+\text { constant }\right]\right\} .
$$

The logarithmic term comes entirely from $\chi_{d e}$. It was first calculated in Ref. (6) and ( 5). $\chi_{e}$ contributes only to the constant in Eq. (2.12). [See Eq. (B2) of Ref. ( (8).]

Note, from Eq. (2.9), that the quantity in brackets in Eq. (2.12) may be written $\lambda_{\text {eff }}(r)+$ $\lambda_{\text {eff }}(r)^{2}+$ constant, to $O\left(\lambda^{2}\right)$. This expression exhibits an infrared divergence at large $r$. That is, for sufficiently large $r, r>\xi_{K}$, the $O\left(\lambda^{2}\right)$ term exceeds the $O(\lambda)$ term. Note however, that this correction term is at least finite as $T \rightarrow 0$. ie. it is $\lambda_{\mathrm{eff}}(r)$ that appears, not $\lambda_{\text {eff }}(T)$. Thus, at least to $O\left(\lambda^{2}\right)$, a finite $r$ is acting like a cut-off on the infrared divergences of perturbation theory. It is an important question whether or not this persists to higher orders in perturbation theory. ie., is perturbation theory valid for $r \ll \xi_{K}$ even for $T \ll T_{K}$, 
with the actual expansion parameter being $\lambda_{\text {eff }}(r)$ ? Based on an examination of higher order terms Gan 1 has argued this not to be the case. He claims that higher order terms diverge as $T \rightarrow 0$ for non-zero $r$ and that it is therefore necessary to have $T \gg T_{K}$ for perturbation theory to be valid. [This point will be examined in detail in Ref. (20).] In this case, it is probably more useful to rewrite Eq. (2.12) in terms of $\lambda_{\text {eff }}(T)$. To $O\left(\lambda^{2}\right)$ :

$$
\chi(r)-\frac{\rho}{2}=\frac{\cos 2 k_{F} r}{32 \pi r^{3} T}\left\{\lambda_{e f f}(T)+\lambda_{e f f}(T)^{2}\left[\ln \left(r T / v_{F}\right)+\text { constant }\right]\right\},
$$

using $E_{F} / k_{F} \approx v_{F}$.

At very low temperatures and large distances, $T \ll T_{K}, r \gg \xi_{K}$, we expect $\chi(r)$ to be determined by the zero-energy fixed point. Within the local Fermi liquid theory ${ }^{\mathrm{B}}$ of this fixed point we can then estimate $\chi(r)$. The zero-energy fixed point corresponds to a screened impurity which just acts as a potential scatterer for the low energy electronic degrees of freedom, with a phase shift of $\pi / 2$ at the Fermi energy. The local susceptibility of a potential scatterer follows directly from the formula for Friedel oscillations in the electron density, $n(r)$, with an s-wave scatterer and a $\pi / 2$ phase shift. For $k_{F} r \gg 1$ :

$$
n(r)=n_{0}-\frac{1}{2 \pi^{2} r^{3}} \cos \left[2 k_{F} r+\pi / 2\right] .
$$

Noting that a magnetic field, $H$, simply shifts the chemical potential by $\pm g \mu_{B} H / 2$ for spin down or spin up electrons, we obtain:

$$
\chi(r, T)=\frac{1}{4 v_{F}} \frac{d n}{d k_{F}}=\frac{\rho}{2}+\frac{1}{4 \pi^{2} v_{F} r^{2}} \cos \left(2 k_{F} r\right) .
$$

Note that $\chi(r, T)$ is longer-range at low $\mathrm{T}$ after the screening cloud has formed, $\chi \propto 1 / r^{2}$, than at higher $\mathrm{T}$ before it has formed, $\chi \propto 1 / r^{3}$, (Eq. (2.5)). An analogous result occurs in spin chain systems21.

Corrections to Eq. (2.15) can be derived by doing perturbation theory in the leading irrelevant operator. Part of the leading correction can be obtained by considering a fielddependence of the phase shift::

$$
\delta^{\sigma}=\pi / 2+\sigma h c / T_{K},
$$


where $c$ is a dimensionless constant of $O(1)$. Generalizing the Friedel oscillation formula of Eq. (2.14), the local density of spin- $\sigma$ electrons becomes:

$$
n_{\sigma}(r)=\frac{n_{0}}{2}-\frac{1}{4 \pi r^{3}} \cos \left[2 k_{F}^{\sigma}(h) r+\delta^{\sigma}(h)\right] .
$$

Upon differentiating with respect to $h$ to obtain the local susceptibility, we now obtain an additional term:

$$
\delta \chi=\frac{c}{4 \pi^{2} r^{3} T_{K}} \cos \left(2 k_{F} r\right) .
$$

Note that this term drops off more rapidly with $r$ than the term in Eq. (2.15) obtained from differentiating $k_{F}^{\sigma}(h)$ and is smaller for $r \gg \xi_{K}$. A very similar Fermi liquid calculation of $\chi(r)$, in the Anderson model, was performed in Ref. 13. However, this calculation effectively ignored the field dependence of $k_{F}^{\sigma}$, and hence only obtained the subdominant term of Eq. (2.18), not the leading term of Eq. (2.15). Explicitly, in Eq. (2.10) of Ref. 13, the free electron Green's function, $F_{r}\left(i \omega_{l}\right)$ must be evaluated in a finite magnetic field. Taking this into account, we obtain our expression, Eq. (2.15).

According to Fermi liquid theory, the impurity susceptibility, $\chi_{\mathrm{imp}}$, defined in Eq. (2.4) is $O\left(1 / T_{K}\right)$. This appears to arise from a short-range part of $\chi(r)$ which does not oscillate at wave-vector $2 k_{F}$. However, since the impurity has been "integrated out" to obtain the Fermi liquid theory, it is difficult to ascertain how much of $\chi_{\text {imp }}$ comes from $\chi(r)$ and how much comes from the impurity self-correlation function, in Eq. (2.4). It is also difficult to tell whether the contribution, if any, from $\chi(r)$ has a range of $O\left(\xi_{K}\right)$ or only of $O\left(1 / k_{F}\right)$, because the cut-off has been reduced to $O\left(1 / \xi_{K}\right)$ to obtain the Fermi liquid theory.

We now wish to formulate a scaling hypothesis for $\chi(r, T)$ which we expect to be valid at arbitrary $r$ and $T$ in the scaling region, $r \gg a, T \ll E_{F}$. For this purpose it is very convenient to use the relativistic one-dimensional formulation of the Kondo problem. [Ref. 22 The mapping to one-dimension is exact for pure s-wave scattering. The use of a reduced bandwidth and linear dispersion relation which leads to the relativistic model is expected to be valid in the scaling region. The three dimensional electron field is expanded in spherical 
harmonics and then the s-wave part is written in terms of left and right moving components (ie. incoming and outgoing):

$$
\psi(\mathbf{x})=\frac{1}{2 \sqrt{2} \pi r}\left[e^{-i k_{F} r} \psi_{L}(r)-e^{i k_{F} r} \psi_{R}(r)\right]+\text { higher harmonics. }
$$

The left and right-moving fields, defined on $r>0$ obey the boundary condition:

$$
\psi_{L}(0)=\psi_{R}(0)
$$

We may flip the right-moving field to the negative axis, so that we work with left-movers only defined on the entire real axis:

$$
\psi_{L}(-x) \equiv \psi_{R}(x)
$$

The one-dimensional Hamiltonian can be written:

$$
H=v_{F} \int_{-\infty}^{\infty} d r \psi_{L}^{\dagger}(r)(i d / d r) \psi_{L}(r)+v_{F} \lambda \psi_{L}^{\dagger}(0) \frac{\boldsymbol{\sigma}}{2} \psi_{L}(0) \cdot \mathbf{S}_{\mathrm{imp}}
$$

$\chi-\rho / 2$ can be expanded in spherical harmonics; only the s-wave harmonic is non-zero. This can be written in terms of one-dimensional uniform and $2 k_{F}$ susceptibilities:

$$
\chi-\rho / 2=\frac{1}{8 \pi^{2} r^{2}}\left[\chi_{\mathrm{un}}+\left(e^{2 i k_{F} r} \chi_{2 k_{F}}+\text { c.c. }\right)\right]
$$

where c.c. denotes complex conjugate and:

$$
\begin{aligned}
\chi_{u n}(r, T) & \equiv(1 / T)<\left[\psi_{L}^{\dagger}(r) \frac{\sigma^{z}}{2} \psi_{L}(r)+\psi_{L}^{\dagger}(-r) \frac{\sigma^{z}}{2} \psi_{L}(-r)\right] S_{T}^{z}> \\
\chi_{2 k_{F}}(r, T) & \equiv(1 / T)<\psi_{L}^{\dagger}(r) \frac{\sigma^{z}}{2} \psi_{L}(-r) S_{T}^{z}>.
\end{aligned}
$$

Here $\mathbf{S}_{T}$ is the total spin in the one-dimensional theory:

$$
\mathbf{S}_{T} \equiv \mathbf{S}_{\mathrm{imp}}+\frac{1}{2 \pi} \int_{-\infty}^{\infty} d r \psi_{L}^{\dagger}(r) \frac{\boldsymbol{\sigma}}{2} \psi_{L}(r)
$$

$\chi_{2 k_{F}}$ can be shown to be real using particle-hole symmetry. This follows since under particle-hole symmetry:

$$
\psi_{L}(r) \rightarrow \sigma^{y} \psi_{L}^{\dagger}(r)
$$


and hence:

$$
\begin{aligned}
\mathbf{S}_{T} & \rightarrow \mathbf{S}_{T} \\
\psi_{L}^{\dagger}(r) \boldsymbol{\sigma} \psi_{L}(-r) & \rightarrow \psi_{L}^{\dagger}(-r) \boldsymbol{\sigma} \psi_{L}(r)=\left[\psi_{L}^{\dagger}(r) \boldsymbol{\sigma} \psi_{L}(-r)\right]^{\dagger} .
\end{aligned}
$$

When particle-hole symmetry is broken, as it is for a realistic Hamiltonian, we expect $\chi_{2 k_{F}}$ to have a phase, $\theta$, which is non-zero but constant in the scaling region. This can be seen from spin-charge separation in the one-dimensional formulation of the Kondo problem. The Kondo interaction, which produces the non-trivial scaling behavior, occurs entirely in the spin sector. In the absence of particle-hole symmetry there is a marginal potential scattering term, $-\theta \psi_{L}^{\dagger}(0) \psi_{L}(0)$, which is a pure charge operator. Upon bosonizing, this is linear in the charge boson and hence doesn't renormalize (is exactly marginal). We can write $\chi_{2 k_{F}}$ as a product of spin and charge correlation functions. The charge correlation function just contributes a constant factor $e^{i \theta}$ to $\chi_{k_{F}}$.

In the lowest two orders of perturbation theory, discussed above, the function $\chi_{\text {un }}$ vanishes at $r \gg 1 / k_{F}$. In fact, it is possible to prove that this happens to all orders in perturbation theory ${ }^{20}$ This is also consistent with Fermi liquid theory, given the uncertainties in that theory, discussed above, about the origin of $\chi_{\mathrm{imp}}$.

We expect the one-dimensional local susceptibility to obey scaling in the following sense. After extracting a factor of $1 / v_{F}, \chi_{2 k_{F}}$ could, in principle depend on three dimensionless variables, which can we taken to be, $r T / v_{F}, \lambda$ and $D / T$. Here $D$ is the effective bandwidth in the one-dimensional theory, a quantity of $O\left(E_{F}\right)$. The scaling hypothesis asserts that the bare coupling constant, $\lambda$ and $D / T$ do not appear independently but only in the combination making up the renormalized coupling constant, $\lambda_{\text {eff }}(T)$. The dependence on $\lambda_{\text {eff }}(T)$ may be exchanged for a dependence on $T / T_{K}$. To see that these two quantities are related, note, from Eqs. (2.9), and (2.10), valid at weak $\lambda_{\mathrm{eff}}$,

$$
\exp \left[\frac{1}{\lambda_{\text {eff }}(T)}\right]=T / T_{K} .
$$

In the intermediate to strong coupling region, the value of $\lambda_{\text {eff }}$ is non-universal, ie. ambiguous. In this regime it is better to use $T / T_{K}$ as a measure of the dimensionless effective 
coupling. This is in accord with the idea that $1 / T_{K}$ is the coupling constant for the leading irrelevant operator at the low-temperature fixed point. As usual, we multiply by the effective cut-off, $T$, to form the dimensionless coupling constant. Thus we write the scaling hypothesis as:

$$
\chi_{2 k_{F}}=\frac{1}{v_{F}} f\left(r T / v_{F}, T / T_{K}\right)
$$

where $f$ is a real universal scaling function. Using Eq. (2.23) the equivalent statement for the three-dimensional susceptibility is:

$$
\chi-\rho / 2=\frac{1}{8 \pi^{2} v_{F} r^{2}} \cos \left(2 k_{F} r\right) f\left(r T / v_{F}, T / T_{K}\right) . \quad\left(r \gg 1 / k_{F} ; T, T_{K} \ll E_{F}\right)
$$

As we will see below, this is consistent with what is known about the behavior at $T \gg T_{K}$ from perturbation theory and $T \ll T_{K}$ and $r \gg \xi_{K}$ from the local Fermi liquid description of the critical point. Note that this scaling hypothesis does imply the existence of the large screening cloud, since if $T \leq T_{K}$, the length scale over which $\chi(r)$ varies is at least $\xi_{K}$ (apart from the $2 k_{F}$ oscillations and the short-range part). In fact, this scaling hypothesis is perhaps the best definition of what it means to have a screening cloud. Note that this scaling form does not include any anomalous dimension. We expect this to be absent since $\mathbf{S}_{\text {tot }}$ is conserved. The more general case, with unequal gyromagnetic ratios for electrons and impurity, involves a non-conserved operator. This will be discussed in Ref. ( 20).

The known results from perturbation theory and Fermi liquid theory, Eq. (2.5), (2.13), (2.15) and (2.18), are all consistent with this scaling hypothesis and imply certain limiting forms for the scaling function. Eq. (2.5) implies that

$$
f(x, y) \rightarrow \frac{\pi^{2}}{2 \sinh (2 \pi x) \ln y},
$$

for $y \ll 1$. Eq. (2.13) gives a higher order correction in $1 / \ln y$ to $\mathrm{f}$, when $x$ is also small. Eq. (2.15) and (2.18) imply that, for $y \ll 1$ and $x / y \gg 1$,

$$
f(x, y) \rightarrow 2+\operatorname{constant} \cdot \frac{y}{x}
$$


The function $f$ in the regime $y \leq 1, x / y=r / \xi_{K} \leq 1$ is of special interest. It describes the interior of the screening cloud at low T. One might naively suppose that a small $r \ll \xi_{K}$ would also cut off the renormalization of the effective coupling so that deep inside the screening cloud we recover weak-coupling behavior (for weak bare coupling) even at low $T$. As mentioned above, Gan ${ }^{\text {l }}$ has argued, based on higher order perturbative calculations, that this is not the case. It follows that the scaling function would be non-trivial in this region.

Now let us consider the experiments of Boyce and Slichter on Fe doped Cu. They measured what they interpreted as the Knight shift from 5 different shells of $\mathrm{Cu}$ atoms at distances up to 5th nearest neighbor. We note that for the $\mathrm{Cu}$ fcc lattice, assuming the $\mathrm{Fe}$ impurities occupy $\mathrm{Cu}$ lattice sites, the fifth nearest neighbor is at a distance of $\sqrt{6} a \approx 2.4 a$ where $a$ is the nearest neighbor separation. The measurements were taken from $\mathrm{T}=300 \mathrm{~K}$ down to well below what is believed to be the Kondo temperature of $29 \mathrm{~K}$. They found the factorized form:

$$
\chi(r, T)=\frac{f(r)}{T+T_{K}},
$$

for some rapidly varying function $f(r)$ (which, in fact, changes sign over the small range of $\mathrm{r}$ considered). Note that all measurements are taken in the regime $r T / v_{F} \ll 1, r T_{K} / v_{F} \ll 1$. In fact the values of $r$ are so small that it is unclear whether the scaling form of Eqs. (2.30) holds at all. In particular, the short-range part of $\chi$ may be contributing. If we assume $r$ is large enough that this can be ignored, and the scaling form holds, then we may consider

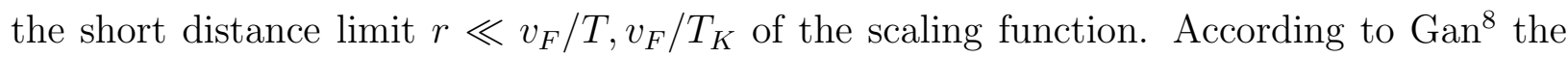
behavior of $f(x, y)$ is non-trivial in the lower temperature range of the experiment, $y \ll 1$, $x / y \ll 1$. ie., we do not know the behavior of the scaling function at low $T$ deep inside the screening cloud. It is possible that $f(x, y)$ exhibits an approximately factorized form for $x / y \ll 1$ and all $y:$

$$
f(x, y) \approx \frac{f(x / y)}{y+1} ? ?
$$

This behavior would explain the experimental results. Note that such factorization could not also occur at large $\mathrm{r}, r \gg \xi_{K}$ if our assumed scaling and asymptotic behaviors are correct. In 
this region, the $1 / r^{3}$ behavior at $T \gg T_{K}$ crosses over to $1 / r^{2}$ at $T \ll T_{K}$. More experiments at larger $r$ could clarify the situation. Experiments in the region $1 / k_{F} \ll r \ll \xi_{K}$ may be feasible. These would probe the short-distance part of the scaling function. A full study of the scaling function would require going out to values of $r \geq \xi_{K}$. One point to bear in mind is that, assuming the existence of a large screening cloud, the average impurity separation is much less than $\xi_{K}$ so inter-impurity interactions may be playing a large role. This may make experimental observation of the screening cloud very difficult, at least until a better understanding of the effect of inter-impurity interactions is obtained.

\section{TIGHT BINDING MODEL}

In order to apply the density matrix renormalization group (DMRG) method we rewrite Eq. (2.1) in real space. This is the standard $s-d$ Kondo model. The model is described by a tight binding Hamiltonian coupled to a $s=1 / 2$ impurity spin, $\mathbf{S}_{\text {imp }}$.

$$
H=-t \sum_{i=1}^{L-1}\left(\psi_{i}^{\dagger \alpha} \psi_{i+1, \alpha}+\psi_{i+1}^{\dagger \alpha} \psi_{i, \alpha}\right)+H_{K}
$$

Here $H_{K}$ describes the coupling to the impurity spin. For the bulk of our results we consider a single $s=1 / 2$ impurity spin at the left end of an otherwise open chain. In this case the coupling to the impurity spin described by $H_{K}$ takes the form

$$
\begin{aligned}
H_{K} & =J \mathbf{S}_{\mathrm{imp}} \cdot \psi_{1}^{\dagger \alpha} \frac{\boldsymbol{\sigma}_{\alpha}^{\beta}}{2} \psi_{1}^{\beta} \\
& =\frac{1}{2} J\left(\left(\psi_{1 \uparrow}^{\dagger} \psi_{1 \uparrow}-\psi_{1 \downarrow}^{\dagger} \psi_{1 \downarrow}\right) S_{\mathrm{imp}}^{z}+\psi_{1 \uparrow}^{\dagger} \psi_{1 \downarrow} S_{\mathrm{imp}}^{-}+\psi_{1 \downarrow}^{\dagger} \psi_{1 \uparrow} S_{\mathrm{imp}}^{+}\right) .
\end{aligned}
$$

We also briefly consider the case of two $s=1 / 2$ impurities located at either end of the chain, correspondingly $H_{K}$ becomes:

$$
H_{K}=J \mathbf{S}_{1} \cdot \psi_{1}^{\dagger \alpha} \frac{\boldsymbol{\sigma}_{\alpha}^{\beta}}{2} \psi_{1}^{\beta}+J \mathbf{S}_{L} \cdot \psi_{L}^{\dagger \alpha} \frac{\boldsymbol{\sigma}_{\alpha}^{\beta}}{2} \psi_{L}^{\beta}
$$

In all our results below we have used $t=1$. 


\section{NUMERICAL METHOD}

We use the density matrix renormalization group (DMRG) method as developed by S. R. White and R. M. Noack23. For a detailed explanation of the method we refer the reader to Ref. 23. The method is centered around calculating the density matrix and its corresponding eigenvalues and eigenvectors for two appropriately defined parts of the total system. Usually this is done by simply considering the system as having a left and a right part. The eigenvalues of the density matrices can then be interpreted as the probability for the subsystem to be in an eigenstate of the density matrix given the constraint that the total system is in a fixed "pure" eigenstate (usually the ground-state). For stable fixed points

of the DMRG method it can be shownet that states in the thermodynamic limit are well represented by "matrix product ground states" 22 27.

In an iteration the density matrices for each half of the system contain $4 \times m$ states (including the $S=1 / 2$ impurity spin). Of these states $m$ are kept to start the next iteration. We have used $m$ in the range 128-200. It is also extremely useful to use all the symmetries of the original Hamiltonian and constrain the whole calculation to a subspace defined by suitable quantum numbers. This increases the precision of the method dramatically. For the Kondo Hamiltonian that we consider here we have in addition to the total z-component of the spin, $S_{T}^{z}$ (including both the impurity and electron spin), and the number of electrons also parity, $P$, for reflection around the midpoint of the chain and, $R P H$ a particle hole symmetry combined with a rotation that changes the sign of the z-component of the spin. The first two are diagonal in the usual product basis and are thus trivial to implement, the $P$ and $R P H$ symmetries are non diagonal and a considerable effort has to be expended to implement these symmetries. The parity $P$, is standard and takes the two values $1,-1$. This is only applicable when we consider two impurities since the one impurity model is not symmetric with respect to a reflection around the middle of the chain. The $R P H$ symmetry is an on-site symmetry and can be used both for the one and two impurity model that we consider. It is defined by 


$$
\begin{aligned}
& R P H: \psi_{j \alpha} \rightarrow(-1)^{j} \psi_{j}^{\dagger \beta}\left(\sigma^{x}\right)_{\beta \alpha} \\
& R P H: \psi_{j}^{\dagger \alpha} \rightarrow(-1)^{j}\left(\sigma^{x}\right)^{\alpha \beta} \psi_{j \beta} \\
& R P H:|0>\rightarrow| F>.
\end{aligned}
$$

Here $\mid 0>$ is the empty state and $\mid F>$ the completely filled state. This is an exact symmetry of the Hamiltonian and it commutes with $P$. We can then specify a state by the four quantum numbers, the filling factor, $S_{T}^{z}, P$, and $R P H$. In the following we shall always work at halffilling, but we chose the remaining quantum numbers so as to select appropriate states. For calculations performed on a system with an impurity at both ends we can use all four quantum numbers, for the case with only one impurity the chain has no longer reflection symmetry around the midpoint of the chain and $P$ is no longer a good quantum number.

The bulk of our results are obtained for the case of only one impurity. In order to obtain optimal precision it is necessary to use a combination of the so called "infinite" and "finite" length DMRG method\$23. At each step of the infinite chain method a complete run of the finite length method is performed. This is done in the following way. We start by considering a 2 site system in addition to the impurity spin. Using the infinite lattice method we generate a 4 site system with matrices representing the impurity spin plus the 2 left sites of the chain and another matrix representing the two right sites. We denote this by $2_{L}+2_{R}$, where the impurity spin is included in the matrix $2_{L}$. The finite system method is then used to arrive at a system consisting of $1_{L}+3_{R}$ where the exact $8 \times 8$ matrix is used for the impurity spin and first site for the matrix $1_{L}$. At this step the expectation values $<S_{\mathrm{imp}}^{z}>$ and $<S_{1}^{z}>$ are calculated since these matrices are known exactly. The finite lattice method is then used to generate $2_{L}+2_{R}$ and $3_{L}+1_{R}$, at each step calculating the expectation value of the electron spins at the sites where the matrices are known exactly, i.e. at sites 2,3 and 3,4 respectively. Then we use the finite system method to generate $3_{L}+3_{R}$ and the whole procedure is repeated. For large chain lengths this is exceedingly slow, but we have been able to treat chain lengths of up to 50 sites. This method is exact out to the point where the matrices $N_{L}$ and $N_{R}$ have to be truncated and it has the great 
merit of yielding the same precision for the expectation value of the spin components in the middle and at the end of the chain. This latter point is essential to our analysis of how the impurity spin is screened at each chain length.

In all cases below we shall take $t=1$ and we always take $L$ even. Furthermore, we shall usually work in the ground-state subspace which for 1 impurity is given by the quantum numbers, $S_{T}^{z}=1 / 2, R P H=1$. For 2 impurities the ground-state subspace is defined by, $S_{T}^{z}=0, R P H=1$, and $P=-1$.

\section{FREE CHAIN}

Let us first consider the tight binding model in the absence of any impurity.

$$
H_{\text {free }}=-t \sum_{i=1}^{L-1}\left(\psi_{i}^{\dagger \alpha} \psi_{i+1, \alpha}+\psi_{i+1}^{\dagger \alpha} \psi_{i, \alpha}\right) .
$$

Here the subscript "free" denotes the free chain. Since we shall be concerned mainly with free boundary conditions for the chain we introduce two "phantom sites", 0 and $L+1$, where we require that $\psi(0)=\psi(L+1)=0$. This model can be solved by transforming to Fourier space and we obtain, in units of the lattice spacing, $a$,

$$
H_{\text {free }}=-2 t \sum_{k} \cos (a k) \psi_{k}^{\dagger \alpha} \psi_{k, \alpha}
$$

where the allowed values for the wave vector, $\mathrm{k}$, are

$$
k=\frac{\pi n}{L+1}, \quad n=1 \ldots L
$$

and thus $0<k<\pi$. We have now essentially two decoupled Fermi seas for up and down spin electrons respectively. The energy is then given by the expression

$$
E_{\text {free }}=-2 t\left(\sum_{k^{\uparrow}}^{k_{F}^{\uparrow}} \cos \left(a k^{\uparrow}\right)+\sum_{k \downarrow}^{k_{F}^{\downarrow}} \cos \left(a k^{\downarrow}\right)\right) \text {. }
$$

Here $k_{F}^{\uparrow}, k_{F}^{\downarrow}$ denotes the Fermi wave vector for up and down spin electrons. We can now choose a simple representation for the field operators. 


$$
\psi_{j}=\sqrt{\frac{2}{L+1}} \sum_{k} \sin (k j) a_{k}, \quad \psi_{j}^{\dagger}=\sqrt{\frac{2}{L+1}} \sum_{k} \sin (k j) a_{k}^{\dagger} .
$$

We note that with this definition the field operators obey the commutation relations $\left\{\psi_{j}, \psi_{j}^{\dagger}\right\}=\delta_{j, l}$

This solution leads to the interesting fact that for a chain with an odd number of sites at exactly half filling, i.e. one electron per site, the magnetization per site (the expectation value of the z-component of the electron spin, $\left\langle S_{j}^{z}\right\rangle$, is given by the following expression

$$
\begin{aligned}
<S_{j}^{z}> & =\frac{1}{2}<\psi_{j}^{\uparrow \dagger} \psi_{j}^{\uparrow}-\psi_{j}^{\downarrow \dagger} \psi_{j}^{\downarrow}> \\
& =\frac{1}{L+1} \sin ^{2} \frac{\pi j}{2}
\end{aligned}
$$

Thus the on-site magnetization, $\left\langle S_{j}^{z}>\right.$, is non-zero only on odd sites, an artifact due to the open boundary conditions. This result can be reproduced by the DMRG method.

For an even length chain, at half-filling, the on-site magnetization is always zero in the ground-state where $S_{T}=0$, since the expectation value of the electron spin is zero at every site due to rotation symmetry. However an excited state with $S_{T}=1$ will have unpaired

spin up electrons in the states with $k=L \pi /(2 L+2),(L+2) \pi /(2 L+2)$. Redoing the above calculation above for an even length chain in the $S_{T}=1$ state we obtain:

$$
<S_{j}^{z}>=\frac{1}{L+1}\left[\sin ^{2} \frac{L \pi j}{2(L+1)}+\sin ^{2} \frac{(L+2) \pi j}{2(L+1)}\right] .
$$

Equivalent results can be obtained for higher excited states.

\section{WEAK COUPLING PERTURBATION}

$$
\text { A. }<S_{j}^{z}>
$$

By considering the term $H_{K}$ in Eq. (3.1) as a perturbation we can do first order perturbation theory in $(J / t)$. We start with a system at half filling with an even number of sites $L$ and we shall take the total z-component of the spin to be $1 / 2, S_{T}^{z}=1 / 2$. The impurity spin is at the far end of the chain at site 1. Here we consider only one impurity described 
by $H_{K}$ in Eq. (3.2). Since we are only considering first order perturbation theory only the term involving $S^{z}$ in $H_{K}$ will contribute. Thus, we have for the unperturbed ground-state

$$
\phi_{0}=\left|\phi>_{\text {free }} \times\right| \uparrow>.
$$

Here $\mid \phi>_{\text {free }}$ is the half-filled sea described in the preceding section and $\mid \uparrow>$ denotes the spin up state of the impurity spin. The first order perturbation to the wave function is

$$
\left|\phi_{1}>=\frac{1-P}{E_{0}-H_{\text {free }}} \frac{J}{4}\left(\psi_{1}^{\dagger \uparrow} \psi_{1}^{\uparrow}-\psi_{1}^{\dagger \downarrow} \psi_{1}^{\downarrow}\right)\right| \phi>_{\text {free }} \times \mid \uparrow>.
$$

Here $P$ is the projection operator onto the ground-state Eq. 6.1). When $L$ is even all single particle states with energy $\varepsilon_{k}=-2 t \cos k$ below the Fermi energy at $\varepsilon_{F}=0, k_{F}=\pi / 2$ are filled for the unperturbed chain. With this notation we can rewrite Eq. (6.2) as

$$
\left|\phi_{1}>=\frac{J}{2 L+2} \sum_{k, k^{\prime}, \varepsilon_{k}<0, \varepsilon_{k^{\prime}}>0} \frac{\sin k \sin k^{\prime}}{\varepsilon_{k}-\varepsilon_{k^{\prime}}}\left(a_{k^{\prime} \uparrow}^{\dagger} a_{k \uparrow}-a_{k^{\prime} \downarrow}^{\dagger} a_{k \downarrow}\right)\right| \phi>_{\text {free }} \times \mid \uparrow>.
$$

Thus, the on-site magnetization becomes

$$
<S_{j}^{z}>=\frac{2 J}{(L+1)^{2}} \sum_{k, k^{\prime}, \varepsilon_{k}<0, \varepsilon_{k^{\prime}}>0} \frac{\sin k j \sin k^{\prime} j \sin k \sin k^{\prime}}{\varepsilon_{k}-\varepsilon_{k^{\prime}}} .
$$

If we now set $k^{\prime}=\pi-k^{\prime \prime}$ we see that $\varepsilon_{k^{\prime}}=-\varepsilon_{k^{\prime \prime}}, \sin k^{\prime} j=-(-1)^{j} \sin k^{\prime \prime} j$ and we can therefore rewrite the above equation as

$$
<S_{j}^{z}>=\frac{J}{t} \frac{(-1)^{j}}{(L+1)^{2}} \sum_{n, m=1}^{L / 2} \frac{\sin k_{n} \sin k_{m} \sin k_{n} j \sin k_{m} j}{\cos k_{n}+\cos k_{m}},
$$

where as above we have $k_{n}=\pi n /(L+1)$. For $j \gg 1$ Eq. (6.5) can be analytically evaluated as:

$$
<S_{j}^{z}>\rightarrow \frac{(J / t)}{4 \pi j}(-1)^{j} .
$$

This formula is basically the $1 \mathrm{D}$ version of the RKKY formula, with $1 / r^{3}$ replaced by $1 / r$ for trivial dimensional reasons. Note that this expression only has a staggered part, not a uniform part. The uniform part is $O\left(J^{2}\right)$. It is quite easy to see that the sum over all $\mathrm{j}$ of Eq. (6.5) vanishes exactly. (This is simply a consequence of the fact that the total electron spin of the unperturbed ground-state, with L even, is zero.) 
The above result, Eq. 6.5), can be compared to DMRG results obtained for weak couplings. In Fig. [1] we show results for a 30 site system with one impurity at the left end. The circles denote $<S_{j}^{z}>$ calculated with the DMRG method, while the crosses are Eq. (6.5). The dotted line indicates the asymptotic form Eq. (6.6). The calculation was performed keeping $m=128$ states in the ground-state subspace defined by $R P H=1, S_{T}^{z}=1 / 2$. Clearly there is a very good agreement between the perturbative results, Eq. (6.5), and the DMRG results.

Equation (6.5) can also be compared to results obtained using the DMRG method with two impurities, one at either end of the chain. In Fig. 2 we show the expectation value of the z-component of the electron spin as a function of chain index, j, for a Kondo coupling, $J$, of 0.05 . In this calculation we have a $S=1 / 2$ impurity spin at each end of the chain. The total z-component of the electron and impurity spin was chosen to be $S_{T}^{z}=1$ so as to polarize the two impurity spins as much as possible, $m=150$ states were kept and we fixed the two remaining quantum numbers $R P H=1, P=1$. As usual we work at half filling. The DMRG results are shown as the circles in Fig. 2. In order to compare these results to Eq. (6.5) we must sum the contribution from the impurity at both ends of the chain. If we denote by $S_{1}^{z(1)}$ the result of Eq. (6.5) for the contribution for one impurity spin we obtain

$$
S_{j}^{z(2)}=S_{j}^{z(1)}+S_{L-j+1}^{z(1)} .
$$

This expression is shown as the $\times$ in Fig. 2. As clearly seen in Fig. 2 there is an excellent agreement between the perturbation results and the DMRG results for the chain length, $L=60$, considered.

\section{B. $S_{\mathrm{imp}}^{z}$}

The expectation value of $S_{\mathrm{imp}}^{z}$ can also be evaluated in first order perturbation theory. Using Eq. (6.2) and remembering to include the contributions from the $\mathrm{x}$ and $\mathrm{y}$ parts of Kondo interaction, we obtain: 


$$
<S_{\mathrm{imp}}^{z}>\approx 1 / 2-\left[\frac{J}{(L+1)}\right]^{2} \sum_{\epsilon_{k}<0, \epsilon_{k^{\prime}}>0}\left[\frac{\sin k \sin k^{\prime}}{\epsilon_{k}-\epsilon_{k^{\prime}}}\right]^{2} .
$$

Where we must be careful to include the correction to the ground-state wave-function normalization. In the large L limit, replacing the sum by an integral, the integral has a log divergence. Thus the last term goes like $\ln L$.

In Fig. 3 we show DMRG results for the z-component of the impurity spin as a function

of chain length, $L$. The results shown are for a very weak Kondo coupling, $J=0.05$. One impurity is present at the left end of the chain. The crosses indicate the first order perturbation result, Eq. (6.8), the circles denote DMRG results for the state $S_{T}^{z}=1 / 2, R P H=1$. A good agreement between the perturbative and numerical results is evident.

\section{Correlation Function}

It is also straightforward to obtain the correlation function $<S_{\mathrm{imp}}^{z} S_{j}^{z}>$ to first order in $J / t$. It is simply $1 / 2$ times Eq. (6.7). In Fig. 田 we show DMRG results for $<S_{\mathrm{imp}}^{z} S_{j}^{z}>$ (circles) for a 50 site chain with impurities at both ends. The Kondo coupling is very weak $J=0.05$. Note that only half the chain is shown. The calculation has been performed with $m=150$ states in the subspace $R P H=1, P=1$, and $S_{T}^{z}=1$. The crosses denote the results from Eq. (6.5) multiplied by $1 / 2$. We see an excellent agreement between the DMRG and the perturbative results.

\section{STRONG COUPLING}

\section{A. Energy}

In the $J=\infty$ limit the impurity traps an electron and forms a tightly bound singlet leaving a free chain with $L-1$ sites. For finite $J$ the singlet can be polarized and we can do perturbation theory in the hopping term between the first and second site. We thus take 


$$
\begin{aligned}
& H=J \mathbf{S}_{\mathrm{imp}} \cdot \psi_{i}^{\dagger \alpha} \frac{\boldsymbol{\sigma}_{\alpha}^{\beta}}{2} \psi_{i}^{\beta}+H_{\text {free }}(L-1)+V \\
& V=-t\left(\psi_{1}^{\dagger \alpha} \psi_{2, \alpha}+\psi_{2}^{\dagger \alpha} \psi_{1, \alpha}\right) .
\end{aligned}
$$

Here $H_{\text {free }}$ is the free chain Hamiltonian Eq. (5.1) for $L-1$ sites. In the following we shall regard the hopping term, $V$, between site 1 and 2 as the perturbation.

The strongly bound singlet on the first site of the chain can be excited into any of the seven excited states shown in Fig. 5. The excited states form a quadruplet with energy $3 J / 4$ and a triplet with energy $J$ relative to the ground-state. The perturbation, $V$, has only non-zero matrix elements between the ground-state and the quadruplet. Calculating the partial matrix elements in the impurity part of the Hilbert space we find

$$
\begin{aligned}
& V_{10}=<1|V| 0>=\frac{1}{\sqrt{2}} \psi_{2}^{\dagger \downarrow} \\
& V_{20}=<2|V| 0>=-\frac{1}{\sqrt{2}} \psi_{2}^{\dagger \uparrow} \\
& V_{30}=<3|V| 0>=-\frac{1}{\sqrt{2}} \psi_{2}^{\uparrow} \\
& V_{40}=<4|V| 0>=-\frac{1}{\sqrt{2}} \psi_{2}^{\downarrow} .
\end{aligned}
$$

Following standard second order perturbation theory we can now calculate the energy shift due to the perturbation $V$. If we denote by $|0>| F>$ the unperturbed ground-state composed of the singlet and the free chain we find

$$
\begin{aligned}
\Delta E & =-\frac{4 t^{2}}{3 J} \sum_{\alpha=1}^{4}<F|<0| V|\alpha><\alpha| V|0>| F> \\
& =-\frac{4 t^{2}}{6 J}<F\left|\left\{\psi_{2}^{+\alpha}, \psi_{2 \alpha}\right\}\right| F> \\
& =-\frac{4 t^{2}}{3 J} .
\end{aligned}
$$

We thus find for the total energy for 1 and 2 impurities, respectively,

$$
\begin{aligned}
& E_{1 \text { impurity }}=-\frac{3}{4} J+E_{\text {free }}(L-1)-\frac{4 t^{2}}{3 J} \\
& E_{2 \text { impurity }}=-\frac{6}{4} J+E_{\text {free }}(L-2)-\frac{8 t^{2}}{3 J} .
\end{aligned}
$$

These perturbation results compare favorably with the DMRG results at sufficiently strong coupling. 


\section{B. wave-function}

We can now calculate the wave-function to second order in perturbation theory. We find

$$
|\phi>=| 0>\left|F>-\frac{4 t}{3 J} \sum_{\alpha=1}^{4} V_{\alpha 0}\right| \alpha>\left|F>+\frac{4}{3}\left(\frac{t}{J}\right)^{2} \sum_{\alpha=1}^{4} V_{5 \alpha} V_{\alpha 0}\right| 5>\mid F>+\cdots .
$$

For the calculations we shall consider here these are the only terms that will contribute. We need to calculate $V_{\alpha 5}$. We find for the partial matrix elements in the impurity part of the Hilbert space,

$$
\begin{aligned}
& V_{15}=<1|V| 5>=\frac{1}{\sqrt{2}} \psi_{2}^{\dagger \downarrow} \\
& V_{25}=<2|V| 5>=\frac{1}{\sqrt{2}} \psi_{2}^{\dagger \uparrow} \\
& V_{35}=<3|V| 5>=-\frac{1}{\sqrt{2}} \psi_{2}^{\uparrow} \\
& V_{45}=<4|V| 5>=\frac{1}{\sqrt{2}} \psi_{2}^{\downarrow} .
\end{aligned}
$$

We can now proceed to evaluate the expectation value of $S_{1}^{z}, S_{2}^{z}, S_{\mathrm{imp}}^{z}$ in perturbation theory.

$$
\text { C. } S_{j}^{z}, S_{\mathrm{imp}}^{z}
$$

We begin by considering the z-component of the electron spin on the first site, $S_{1}^{z}$. In the unperturbed system $S_{1}^{z}$ must be zero since it is locked in a singlet with the impurity spin. The first non-zero contribution to $S_{1}^{z}$ is second order in $t / J$. Since

$$
S_{1}^{z}\left|0>=-\frac{1}{2}\right| 5>
$$

we find that only the second term in Eq. (7.6) will contribute and we get

$$
<S_{1}^{z(2)}>=2<F|<0| S_{1}^{z} \frac{4}{3}\left(\frac{t}{J}\right)^{2} \sum_{\alpha=1}^{4} V_{5 \alpha} V_{\alpha 0}|5>| F>=-\frac{4}{3}\left(\frac{t}{J}\right)^{2}<F\left|\psi_{2}^{\dagger \uparrow} \psi_{2}^{\uparrow}-\psi_{2}^{\dagger \downarrow} \psi_{2}^{\downarrow}\right| F>^{(2)}
$$

From this we can derive two results; if the state $\mid F>$ describes an unperturbed chain with $L-1$ sites we find from Eq. (5.6) 


$$
<S_{1}^{z(2)}>=-\frac{8}{3}\left(\frac{t}{J}\right)^{2} \frac{1}{L}
$$

since in that case the matrix element in Eq. (7.9) is simply $2 / L$. We can also consider the case where $\mid F>$ describes a free chain with $L-2$ sites but now in a state with $S_{T}^{z}=1$. This is convenient for comparing with results with 2 impurities where 2 sites will be quenched out. It is in that case convenient to work in the state with $S_{T}^{z}=1$. We then find

$$
<S_{1}^{z(2)}>=-\frac{8}{3}\left(\frac{t}{J}\right)^{2} \frac{1}{L-1}\left[\sin ^{2} \frac{\pi(L-2)}{2(L-1)}+\sin ^{2} \frac{\pi L}{2(L-1)}\right]
$$

In a similar fashion $S_{\text {imp }}^{z(2)}$ can be calculated to second order in $t / J$. We first consider the case with one impurity. Again $S_{\mathrm{imp}}^{z}$ is zero in the unperturbed state. For $S_{\mathrm{imp}}^{z}$ we find

$$
S_{\text {imp }}^{z}\left|0>=\frac{1}{2}\right| 5>
$$

Thus we get the same term as before but with a different sign. However, since $S_{\text {imp }}^{z}$ is nonzero in the quadruplet we get an additional term from the second term in Eq. (7.6). We then have

$$
\begin{aligned}
<S_{\text {imp }}^{z(2)}> & =\frac{16}{9}\left(\frac{t}{J}\right)^{2} \sum_{\alpha=1}^{4}<F\left|V_{0 \alpha} V_{\alpha 0}\right| F><\alpha\left|S_{\text {imp }}^{z}\right| \alpha>+\frac{4}{3}\left(\frac{t}{J}\right)^{2}<F\left|\psi_{2}^{\dagger \uparrow} \psi_{2}^{\uparrow}-\psi_{2}^{\dagger \downarrow} \psi_{2}^{\downarrow}\right| F> \\
& =\frac{20}{9}\left(\frac{t}{J}\right)^{2}<F\left|\psi_{2}^{\dagger \uparrow} \psi_{2}^{\uparrow}-\psi_{2}^{\dagger \downarrow} \psi_{2}^{\downarrow}\right| F>^{(2)}
\end{aligned}
$$

As before we can now obtain the results for the case where $\mid F>$ describes a chain with $L-1$ sites, ie when only one impurity is present.

$$
<S_{\mathrm{imp}}^{z(2)}>=\frac{40}{9}\left(\frac{t}{J}\right)^{2} \frac{1}{L}
$$

And equivalently for the state with $L-2$ sites and $S_{T}^{z}=1$ where we have the 2 impurity case in mind.

$$
<S_{\mathrm{imp}}^{z(2)}>=\frac{40}{9}\left(\frac{t}{J}\right)^{2} \frac{1}{L-1}\left[\sin ^{2} \frac{\pi(L-2)}{2(L-1)}+\sin ^{2} \frac{\pi L}{2(L-1)}\right]
$$

To this order in perturbation theory we have in addition the following equality for the second order contribution to $<S_{2}^{z}>$ : 


$$
<S_{2}^{z(2)}>=-<S_{1}^{z(2)}>-<S_{\mathrm{imp}}^{z(2)}>
$$

Note that for $\left\langle S_{2}^{z}>\right.$ we still have the term $1 / L$ from Eq. (5.6) which we can apply here at strong coupling. These equations are obeyed to a high accuracy at sufficiently high coupling $J$. To illustrate this we show in Fig. 6 results for one impurity with a Kondo coupling of $J=10$ for $L=24$. The calculation is done keeping $m=128$ states with $R P H=1$ and $S_{T}^{z}=1 / 2$. The circles denote the DMRG results for $<S_{j}^{z}>$ and the square denote $<S_{\mathrm{imp}}^{z}>$. The crosses denote the results from Eq. (5.6) for a 23 site chain with the corrections from Eqs. (7.11) and (7.16). The plus is the result for $<S_{\text {imp }}^{z}>$ from Eq. (7.14). From the results in Fig. 6 we find excellent agreement between the above perturbation results and our DMRG results at strong coupling.

We note that the fact that $\left\langle S_{j}^{z}>\rightarrow\right.$ constant $/ L$ for $j \gg \xi_{K}$, and thus in a sense is longer range than $\operatorname{RKKY}(1 / \mathrm{r})$, is analogous to the prediction that $\chi(r, T)$ should be longer range when the screening cloud has formed (see discussion below Eq. (2.15)), than when it has not.

\section{D. correlation function}

It is also rather straight forward to calculate the correlation function $<S_{\mathrm{imp}}^{z} S_{j}^{z}>$ to second order in $t / J$. Again the unperturbed result is just zero for $j>1$. Let us first consider the case where $j>2$, in which case we get from Eq. (7.13)

$$
\begin{aligned}
<S_{\mathrm{imp}}^{z} S_{j}^{z}>^{(2)} & =\frac{20}{9}\left(\frac{t}{J}\right)^{2}<F\left|S_{j}^{z}\left(\psi_{2}^{\dagger \uparrow} \psi_{2}^{\uparrow}-\psi_{2}^{\dagger \downarrow} \psi_{2}^{\downarrow}\right)\right| F> \\
& =\frac{10}{9}\left(\frac{t}{J}\right)^{2} G(L-2, j-1) j>2 .
\end{aligned}
$$

Here we consider the case where $\mid F>$ describes a free chain with $L-2$ sites corresponding to two impurities, and $G$ is given by

$$
G(L, j)=<F\left|\left(\psi_{1}^{\dagger \uparrow} \psi_{1}^{\uparrow}-\psi_{1}^{\dagger \downarrow} \psi_{1}^{\downarrow}\right)\left(\psi_{j}^{\dagger \uparrow} \psi_{j}^{\uparrow}-\psi_{j}^{\dagger \downarrow} \psi_{j}^{\downarrow}\right)\right| F>
$$

For the states describing the free chain $G$ can easily be calculated. For the state that we consider here with $L-2$ sites and $S_{T}^{z}=0$, we find 


$$
\begin{aligned}
G(L, j) & =\frac{8}{(L+1)^{2}}\left(\sum_{k<k_{F}} \sin k \sin k j\right)\left(\sum_{k>k_{F}} \sin k \sin k j\right) \\
& =-\frac{8}{(L+1)^{2}}\left(\sum_{k<k_{F}} \sin k \sin k j\right)^{2} .
\end{aligned}
$$

As before we have $k=\pi n /(L+1), n=1 \cdots L$. When $j=2$ we have to be somewhat more

careful. Reanalyzing the two terms in Eq. (7.13) we find that due to the non-commutativity of $S_{2}^{z}$ and $V$ we effectively get a sign change on the first term and thus

$$
<S_{\mathrm{imp}}^{z} S_{2}^{z}>^{(2)}=\frac{2}{9}\left(\frac{t}{J}\right)^{2} G(L-2,1)=\frac{1}{9}\left(\frac{t}{J}\right)^{2} .
$$

Furthermore we must have that

$$
<S_{\mathrm{imp}}^{z} S_{1}^{z}>^{(2)}=-\frac{1}{4}-\frac{\Delta E}{3 J},
$$

for the case where we have only one impurity where $\Delta E$ is given by Eq. (7.4). Except for the first argument to $G$ we note that the above results for the correlation function do not depend in any essential way on whether we consider 1 or 2 impurities.

We illustrate this with a calculation of $<S_{\text {imp }}^{z} S_{j}^{z}>$ for a 30 site chain with an impurity at both ends. We keep $m=150$ states and work in the ground-state subspace with $R H P=$ 1, $P=-1$, and $S_{T}^{z}=0$. We first consider $<S_{\mathrm{imp}}^{z} S_{1}^{z}>$. From the DMRG we find $<$ $S_{\mathrm{imp}}^{z} S_{1}^{z}>=-0.24636$. This can be compared with the result from Eq. (7.21) which gives $<S_{\mathrm{imp}}^{z} S_{1}^{z}>^{(2)}=-.24555$ in good agreement. The results for $j \geq 2$ are shown in Fig. 7. The circles denote the DMRG results and the crosses the results from Eqs. (7.19) and (7.20). Clearly the discrepancy is largest for $j=2,3$. As a function of chain length, $L$, we have observed that this discrepancy decreases. Over all the agreement with the perturbative results is very good.

\section{CROSSOVER REGIME}

Having checked that the DMRG method yields the expected perturbation results in the strong and weak coupling limit we now proceed to study the crossover behavior and the 
scaling predicted by Eqs. (2.29). Since the numerical results are all obtained at $T=0$ for finite systems, where $S_{\text {tot }}^{z}$ is a constant, the role played by $\Delta \chi \equiv \chi(r, T, J)-\rho / 2$ is taken by the the on-site magnetization, $\Delta S \equiv<S_{j}^{z}>$ (expectation value of the z-component of the electron spin at site $j$ ). Hence, a generalized finite size form of Eqs. (2.29) should apply to $<S_{j}^{z}>$ at $T=0$. Such a finite-size form, applicable to the numerical results for finite $L$, is easily obtained; we simply substitute $L$ for the thermal length, $v_{F} / T$. In this way we get $r T / v_{F} \rightarrow r / L$ and $T / T_{K} \rightarrow \xi_{K} / L$. We note that in order to apply Eq. (2.29) which was derived for a susceptibility (Eq. (2.3)), to the on-site magnetization we need to multiply by T. We then obtain:

$$
<S_{j}^{z}>=\frac{1}{L}\left\{\tilde{f}\left(\frac{j}{\xi_{K}}, \frac{L}{\xi_{K}}\right)(-1)^{j}+\widetilde{g}\left(\frac{j}{\xi_{K}}, \frac{L}{\xi_{K}}\right)\right\}
$$

where we have kept the finite-size equivalent of $\chi_{\text {un }}$ as well as $\chi_{2 k_{F}}$. We note that this form assures that $\sum_{j}<S_{j}^{z}>\approx\left(\xi_{K} / L\right) \int d x \tilde{g}\left(x, L / \xi_{K}\right)$ is a function of $\xi_{K} / L$ only.

\section{A. Scaling of $L<S_{j}^{z}>$}

We now proceed to test the above scaling form for the finite systems we have been able to study numerically. We start by considering how the weak and strong coupling results of Sections VI and VII can be cast into a from consistent with Eq. (8.1). First we note that the strong coupling expression Eq. (5.6) obviously obeys the scaling form. However, the asymptotic weak coupling result, Eq. (6.6) doesn't seem to obey the scaling form due to the explicit dependence on $J / t$. Fortunately it is possible to remedy this by noting that $J / t \sim 1 / \ln \left(\xi_{K} / L\right)$. This we see in the following way: We can write our 2 scaling variables as $j / L$ and $\xi_{K} / L$. Alternatively, and perhaps better, we can replace $\xi_{K} / L$ by the effective renormalized dimensionless Kondo coupling at scale $L$. Here we define a dimensionless Kondo coupling as $\lambda \equiv J / t$. Combining Eqs. (2.9) and (2.10) we get using $\Lambda^{-1}=L$ :

$$
\lambda_{e f f}=\frac{1}{\ln \left(\xi_{K} / L\right)}
$$


As $L$ becomes small (ie. approaches 1) $\lambda_{\text {eff }}(L)$ approaches the bare coupling constant, $\lambda$. Thus, for small $L$, and weak coupling, we have, by substituting $1 / \ln \left(\xi_{K} / L\right)$ for $(J / t)$ :

$$
L<S_{j}^{z}>\rightarrow(-1)^{j} \frac{L}{j} \frac{1}{4 \pi \ln \left(\xi_{K} / L\right)},
$$

perfectly consistent with scaling.

First we consider $L<S_{L / 2}^{z}>$. In this case the scaling function should be $\widetilde{g}\left(1 / 2, L / \xi_{K}(J)\right)+(-1)^{L / 2} \widetilde{f}\left(1 / 2, L / \xi_{K}(J)\right)$, and we can suppress the first argument. In addition since we only consider the case where $L / 2$ is even, the scaling relation takes the simpler form $L<S_{L / 2}^{z}>=h\left(L / \xi_{K}\right)$. In Fig. \& we show $L<S_{L / 2}^{z}>$ for the coupling constants $J=0.3,0.4,0.5,0.75,1,1.5,1.8,2,2.5,3,3.5,4,10$ as a function of $L / \xi_{K}$, beginning with $L=2$. The data can be collapsed onto a single curve thus determining $\xi_{K}(J)$ up to a multiplicative constant. If we fix one of the correlation lengths the rest of the correlation lengths are fixed by requiring that the scaling form be obeyed. An excellent data collapse is obtained. All the results in this figure are for the one impurity case in the ground-state subspace $R P H=1, S_{T}^{z}=1 / 2$, with $m=128$ states. In principle it is possible to obtain the Kondo length scale, $\xi_{K}$ as a function of $J$ from this scaling plot. However, since $\xi_{K}$ varies quite rapidly with $J$ one can essentially only obtain qualitative results at weak couplings where $\xi_{K}$ is several thousand lattice spacings or at strong couplings where $\xi_{K}$ is very small. However, for a fair range of intermediate couplings $\xi_{K}$ can be extracted with a reasonable precision. Our results are summarized in Table $\square$. We note that Fig. 目 clearly displays the complete crossover from weak to strong coupling.

In the same manner we can look at $L<S_{j}^{z}>$, but instead of fixing $j / L$ we can fix $L / \xi_{K}(J)$. The scaling form should then be $h\left(j / \xi_{K}(J)\right)$, where we have suppressed the dependence on $L / \xi_{K}(J)$. Using $\xi_{K}(J=2.5)=1.0, \xi_{K}(J=3)=0.6$, such a data collapse is shown in Fig. 9 with $L=30,20$ for $J=2.5,3$, respectively. Thus, $L / \xi_{K} \approx 32$ is kept fixed. Viewing $\xi_{K}(J=2.5)$ and $\xi_{K}(J=3)$ as fixed from the previous analysis this plot contains no free parameters. The collapse is excellent. The same rescaling can also be performed at other couplings. In Fig. 10 we show results for $J=1.5$ and $J=1.8$ with $\xi_{K}(J=1.5) \sim 4.85$ 
and $\xi_{K}(J=1.8) \sim 2.7$, where we use $L=36,20$ for $J=1.5,1.8$ respectively. Again we find an excellent collapse of the data. The complete scaling shown in Figs. 9 and 10 is a highly non-trivial test of the scaling form Eq. (8.1) and the fact that the numerical results clearly follow the scaling form lends strong support to the existence of the Kondo length scale.

\section{B. Scaling of $L<S_{\mathrm{imp}}^{z}>$}

We now turn to a discussion of our results for the expectation value of the impurity spin. In Fig. 11 we show $L<S_{\mathrm{imp}}^{z}>$ as a function of $L$ for $J=0.5,1,1.5,1.8,1.9,2,2.5,3,3.5,4,10$, beginning with $L=2$. As is clearly evident $L<S_{\mathrm{imp}}^{z}>$ approaches a constant in the strong coupling limit. At weak coupling the behavior is consistent with $L<S_{\mathrm{imp}}^{z}>\sim L / 2$ for the values of $L$ accessible. From the results in Fig. 11 we can also check the result Eq. (7.14). For $J=10.0$ we find with the DMRG method that $L<S_{\text {imp }}^{z}>\sim 0.0456$ for large $L$. This can be compared to 0.0444 from Eq. (7.14), in very good agreement.

\section{C. $\xi_{K}$}

In the previous section we obtained numerical results for $\xi_{K}(J)$ by requiring our numerical results to scale. We can now try to fit these results to expressions for $\xi_{K}$ obtained from renormalization group arguments which to first order gives Eq. (2.10). Is this possible ? The first point to realize is that we can only expect the weak coupling RG formula to work for a range of $J$ such that $1 \ll \xi_{K} \ll L$. If $\xi_{K}$ is too small then the coupling constant is too big so low order perturbation theory doesn't work. If $\xi_{K}$ is too big then finite size effects will dominate.

We proceed by obtaining a higher order expression for $\xi_{K}(J)$ than the simple exponential relation Eq. (2.10). To go to one higher order, we first of all need to calculate the renormalized coupling to $O\left[(J \rho)^{2}\right]$, obtained by reducing the effective bandwidth (in momentum space) from $\pi / a$ to $2 / \xi_{0}$, where $\xi_{0}$, is some length scale much bigger than $a$, the lattice spacing. This involves the integral: 


$$
\int_{0}^{\pi / 2 a-1 / \xi_{0}} \frac{d k}{\cos k a} \propto \ln \left[\tan \left(2 \xi_{0} / a\right)\right]
$$

Thus:

$$
\lambda_{0}=J \rho+(J \rho)^{2} \ln \left[\tan \left(2 \xi_{0} / a\right)\right]
$$

Here $\rho$ is the density of states,

$$
\rho=1 / 2 \pi t
$$

Next we need to integrate the $\beta$-function, to third order. The $\beta$-function is 28 :

$$
-d \lambda / d \ln \Lambda=\lambda^{2}-\lambda^{3} / 2
$$

We now integrate this equation, using the bare cut-off, $\Lambda_{0}=1 / \xi_{0}$, the bare coupling $\lambda_{0}$ given above, the renormalized coupling some number c of $O(1)$ and the new cut-off $1 / \xi_{K}$, the inverse correlation length. This gives29.

$$
\xi_{K}=\xi_{0} e^{1 / \lambda_{0}-1 / c} \sqrt{\frac{1-2 / \lambda_{0}}{1-2 / c}} .
$$

This, together with the equation above for $\lambda_{0}$ determine $\xi_{K}$ vs. J to a better accuracy than the simple exponential form. We now have 2 free parameters; c which should be positive and $O(1)$ and $\xi_{0}$ which should be $\gg 1$ but $\ll \xi_{K}$. This form should be valid for the range of $J$ where $1 \ll \xi_{K} \ll L$. The corrections arising from the constant $c$ is presumably only one of several equally important terms. We have included it here to improve the agreement with the numerical results. In the limit $\lambda_{0} \rightarrow 0$ Eq. (8.8) reduces to: $\xi_{K} \propto e^{1 / \lambda_{0}} / \sqrt{\lambda_{0}}$, in agreement with Ref. 30 .

A least square fit of the numerical results for $\xi_{K}(J)$, in Table [1, to the form Eq. 8.8 is shown in Fig. 12. The circles indicate the DMRG results while the solid line is Eq. (8.8) with the fitted parameters $\xi_{0}=1.76, c=0.21$. Taking into account the sizable uncertainty in $\xi_{K}$ at weak coupling the fit works extraordinarily well. 


\section{CONCLUSION}

We have shown that the local susceptibility can be brought into a unified scaling form linking a high temperature RRKY form and a low temperature local Fermi liquid form. In this picture the Knight shift is longer range at low temperatures where the screening cloud has formed, than at high temperatures where it has not. The experimentally observed behavior of the Knight shift 1 can possibly be explained by a factorization of the scaling functions deep inside the screening cloud which is the only region the experiments probe.

Non-trivial tests of the scaling form have been performed by numerically calculating the on-site magnetization at zero temperature for finite systems. In all cases scaling behavior consistent with the proposed form is observed. Both $\left\langle S_{L / 2}^{z}>\right.$ as a function of $L$, and $<S_{j}^{z}>$ as a function of $j$ for fixed $\xi_{K} / J$ clearly show scaling. Numerical estimates of $\xi_{K}(J)$ has been extracted in good agreement with an estimate of $\xi_{K}(J)$ from renormalization group calculations.

\section{ACKNOWLEDGMENTS}

We gratefully acknowledge many useful discussions with J. Gan. We would also like to thank V. Barzykin, W. J. L. Buyers, S. M. Girvin, B. I. Halperin, A. H. MacDonald, N. Prokof'ev, P. C. E. Stamp, C. Varma, E. Wong and A. Zawadowski for helpful comments. ESS is supported by NSF grant number NSF DMR-9416906. This research was supported in part by NSERC of Canada. 


\section{REFERENCES}

${ }^{1}$ K. G. Wilson, Rev. Mod. Phys. 47, 773 (1975); H. R. Krishna-murthy, J. W. Wilkins, and K. G. Wilson, Phys. Rev. B. 21, 1003 (1980); 21, 1044 (1980).

${ }^{2}$ P. W. Anderson, J. Phys. C 3, 2439 (1970).

3 (a) Ph. Nozières, J. Low Temp. Phys. 17, 31 (1974); (b) Ph. Nozières, J. de Phys. 39, 1117 (1978); (c) Ph. Nozières, in Proc. 14th Int. Conf. on Low Temperature Physics, eds. M. Krusius and M. Vuorio, Vol. 5, (North Holland, Amsterdam 1975); (d) Ph. Nozières and A. Blandin, J. Physique 41, 193 (1980).

${ }^{4}$ N. Andrei, Phys. Rev. Lett. 45, 379 (1980); P. B. Wiegmann, Sov. Phys. JETP Lett. 31, 392 (1980). N. Andrei, K. Furuya, and J. H. Lowenstein, Rev. Mod. Phys. 55, 331 (1983).

${ }^{5}$ R.H. Bressmann and M. Bailyn, Phys. Rev. 154, 471 (1967).

${ }^{6}$ M.S. Fullenbaum and D.S. Falk, Phys. Rev. 157, 452 (1967).

${ }^{7}$ H. Keiter, Z. Phys. 223, 289 (1969).

8 J. Gan, J. Phys.: Cond. Mat. 6, 4547 (1994); J. Gan, private communication.

${ }^{9}$ E. Muller-Hartmann, Z. Phys. 223, 277 (1969).

${ }^{10}$ K. Chen, C. Jayaprakash and, H. R. Krishnamurthy, Phys. Rev. B 45, 5368 (1992).

${ }^{11}$ J. E. Hirsch and R. M. Fye, Phys. Rev. Lett. 56, 2521 (1986); J. E. Gubernatis, J. E. Hirsch and D. J. Scalapino, Phys. Rev. B 35, 8478 (1987); R. M. Fye and J. E. Hirsch, Phys. Rev. B 38, 433 (1988).

12 D. Šokčević, V. Zlatić and B. Horvatić, Phys. Rev. B 39, 603 (1989).

${ }^{13}$ H. Ishii, J. Low Temp. Phys. 32, 457 (1978); Prog. Th. Phys. 55, 1373 (1976); Physica B 86-88, 517 (1977).

14 J. Kondo, Solid State Physics 23, 183 (1969). 
${ }^{15}$ A. C. Hewson, The Kondo Problem to Heavy Fermions, Cambridge University Press, Cambridge 1993.

${ }^{16}$ J. P. Boyce and C. P. Slichter, Phys. Rev. Lett. 32, 61 (1974); Phys. Rev. B 13, 379 (1976).

${ }^{17}$ H. Alloul, Phys. Rev. Lett. 35, 460 (1975); For a review see H. Alloul, Physica B 86-88, 449 (1977); A. Narath, in Magnetism Vol V, eds G. T. Rado and H. Suhl, Academic Press, New York 1973, p. 149.

${ }^{18}$ G. Chen and N. Giordano, Phys. Rev. Lett. 66, 209 (1991); M. A. Blachly and N. Giordano, Phys. Rev. B 46, 2951 (1992); J. F. DiTusa, K. Lin, M. Park, M. S. Isaacson, and J. M. Parpia, Phys. Rev. Lett. 68, 678 (1992); V. Chandrasekhar, P. Santhanam, N. A. Penebre, R. A. Webb, H. Vloeberghs, C. Van Haesendonck, and Y. Bruynseraede, Phys. Rev. Lett. 72, 2053 (1994).

${ }^{19}$ O. Újsághy, A. Zawadowski, and B. Gyorffy, preprint 1995.

${ }^{20} \mathrm{~V}$. Barzykin and I. Affleck, in progress.

${ }^{21}$ S. Eggert and I. Affleck, Phys. Rev. Lett. 75, 934 (1995).

${ }^{22}$ See for example I. Affleck and A. W. W. Ludwig, Nucl. Phys. B 360, 641 (1991) appendix A.

${ }^{23}$ S. R. White and R. M. Noack, Phys. Rev. Lett. 68, 3487 (1992); S. R. White, Phys. Rev. Lett. 69, 2863 (1992); Phys. Rev. B 48, 10345 (1993).

${ }^{24}$ S. Östlund and S. Rommer, Phys. Rev. Lett. 75, 3537 (1995).

${ }^{25}$ L. Accardi, Phys. Rep. 77, 169 (1981).

${ }^{26}$ M. Fannes, B. Nachtergaele, and R. F. Werner, Europhys. Lett. 10, 633 (1989); Commun. Math. Phys. 144443 (1992). 
${ }^{27}$ A. Klümper, A. Schadschneider, and J. Zittartz, Europhys. Lett. 24, 293 (1993); C. Lange, A. Klümper, and J. Zittartz, Zeit. Phys. B 96, 267 (1994).

${ }^{28}$ M. Fowler and A. Zawadowski, Sol. State Comm. 9, 471 (1971). A. A. Abrikosov and A. A. Migdal, J. Low. Temp. Phys. 3, 519 (1970).

${ }^{29}$ An analogous calculation can be found in A. Sikkema and I. Affleck, Phys. Rev. B 52, 10207 (1995).

${ }^{30}$ For a review see Ref. 3(c) and G. Grüner and A. Zawadowski, Rep. Prog. Phys. 37, 1497 (1971). 


\section{TABLES}

TABLE I. The values for the Kondo length, $\xi_{K}$, used in Fig. 8 and shown in Fig. 12 (circles).

\begin{tabular}{cc}
\hline \hline $\mathrm{J}$ & $\xi_{K}(J)$ \\
\hline 10.0 & 0.031 \\
4.0 & 0.27 \\
3.0 & 0.6 \\
2.5 & 1.0 \\
2.0 & 2.0 \\
1.8 & 2.7 \\
1.5 & 4.85 \\
1.0 & 23 \\
0.75 & 100 \\
0.5 & 1200 \\
0.4 & 7000 \\
0.3 & 100000 \\
\hline \hline
\end{tabular}




\section{FIGURES}

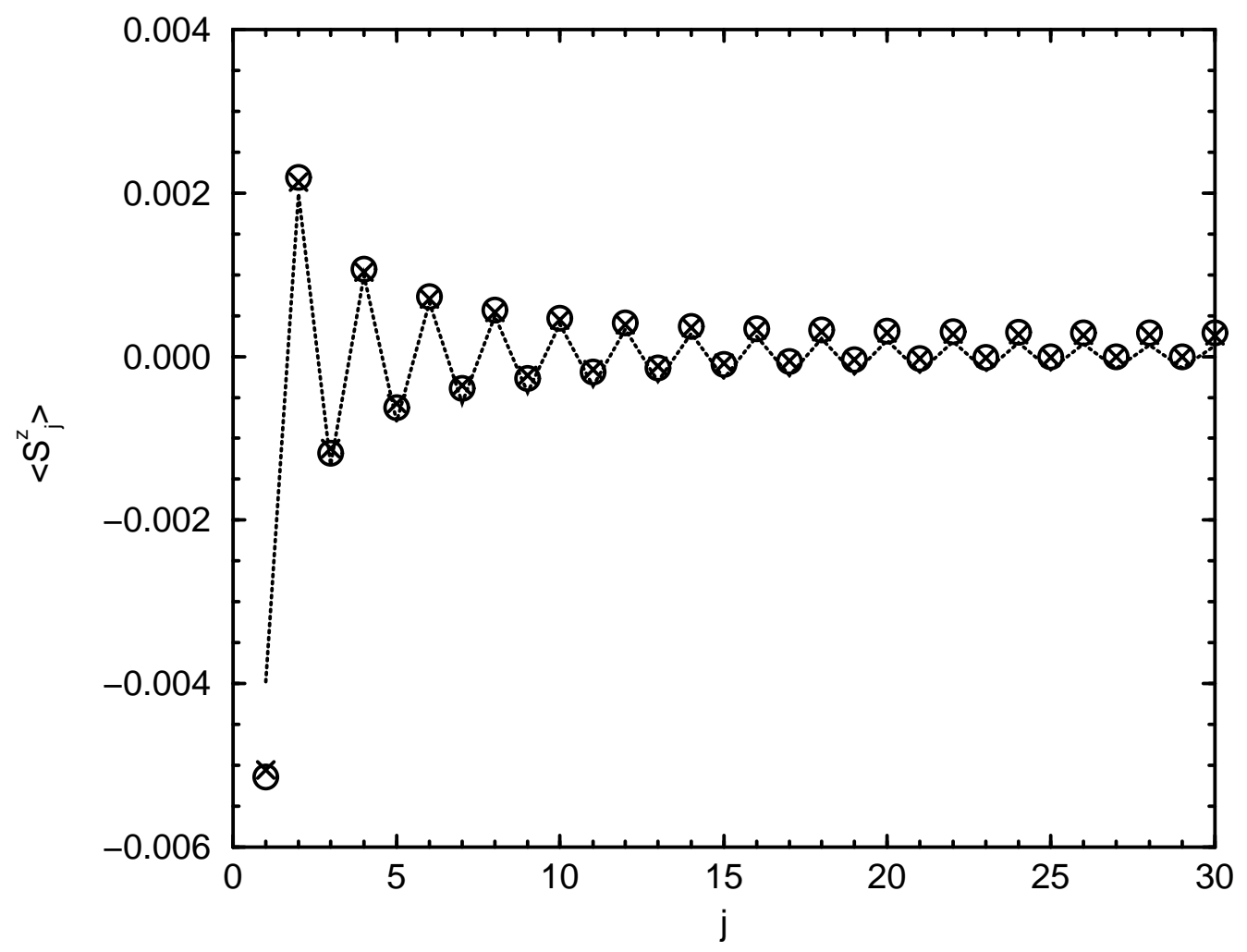

FIG. 1. The z-component of the electron spin as a function of site index $\mathrm{j}$, for a chain of length $L=30$. At the left end of the chain is an $S=1 / 2$ impurity spin. The Kondo coupling is $J=0.05$. The crosses indicate the first order perturbation result, Eq. (6.5), the circles denote DMRG results for the state $S_{T}^{z}=1 / 2, R P H=1 . m=128$ states were kept. The dotted line is the asymptotic result Eq. (6.6). 


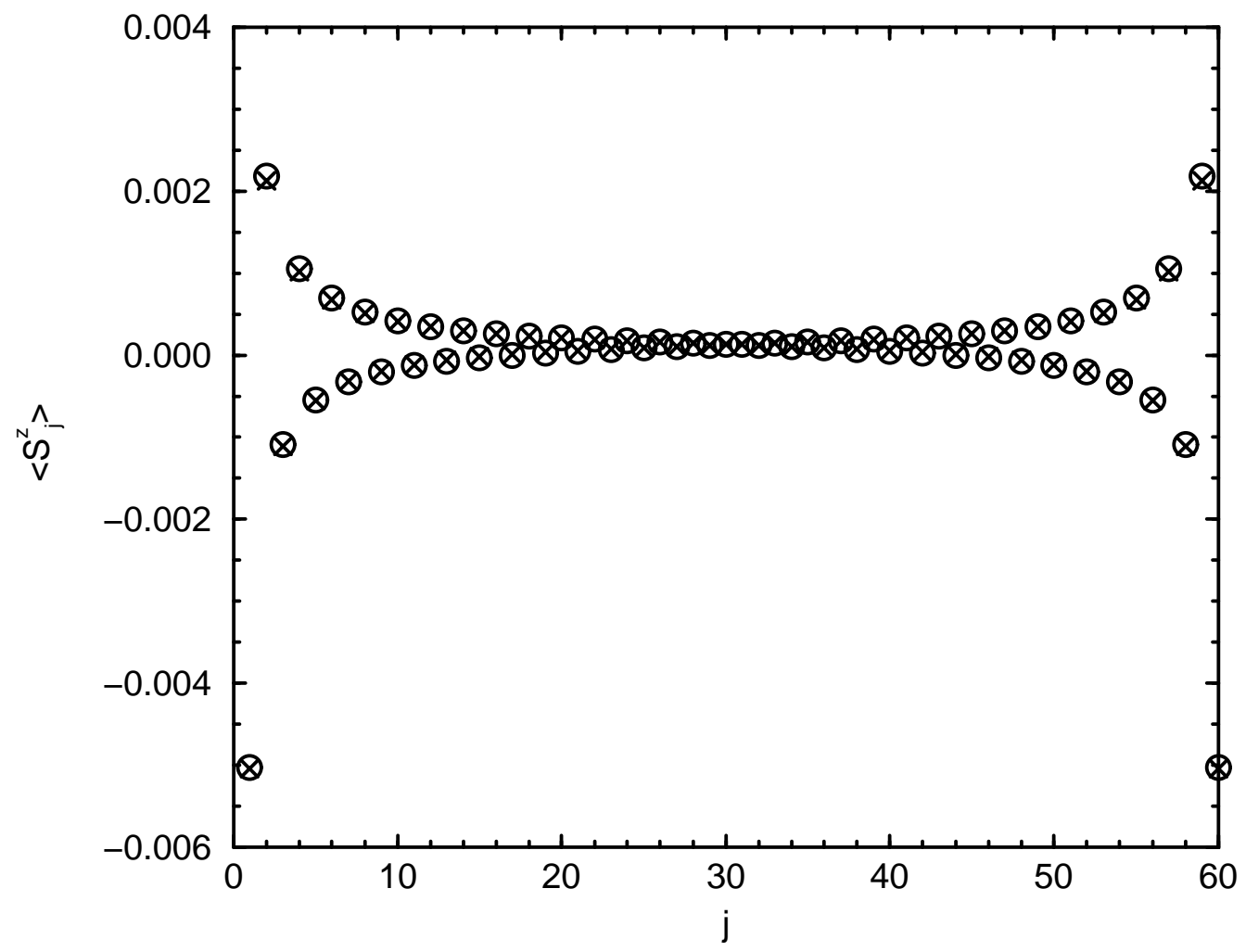

FIG. 2. The z-component of the electron spin as a function of site index $\mathrm{j}$, for a chain of length $L=60$. Impurity spins are present at both ends of the chain. The Kondo coupling is $J=0.05$. The crosses indicate the first order perturbation result, Eq. 6.7), the circles denote DMRG results for the state $S_{T}^{z}=1, R P H=1, P=1$. 


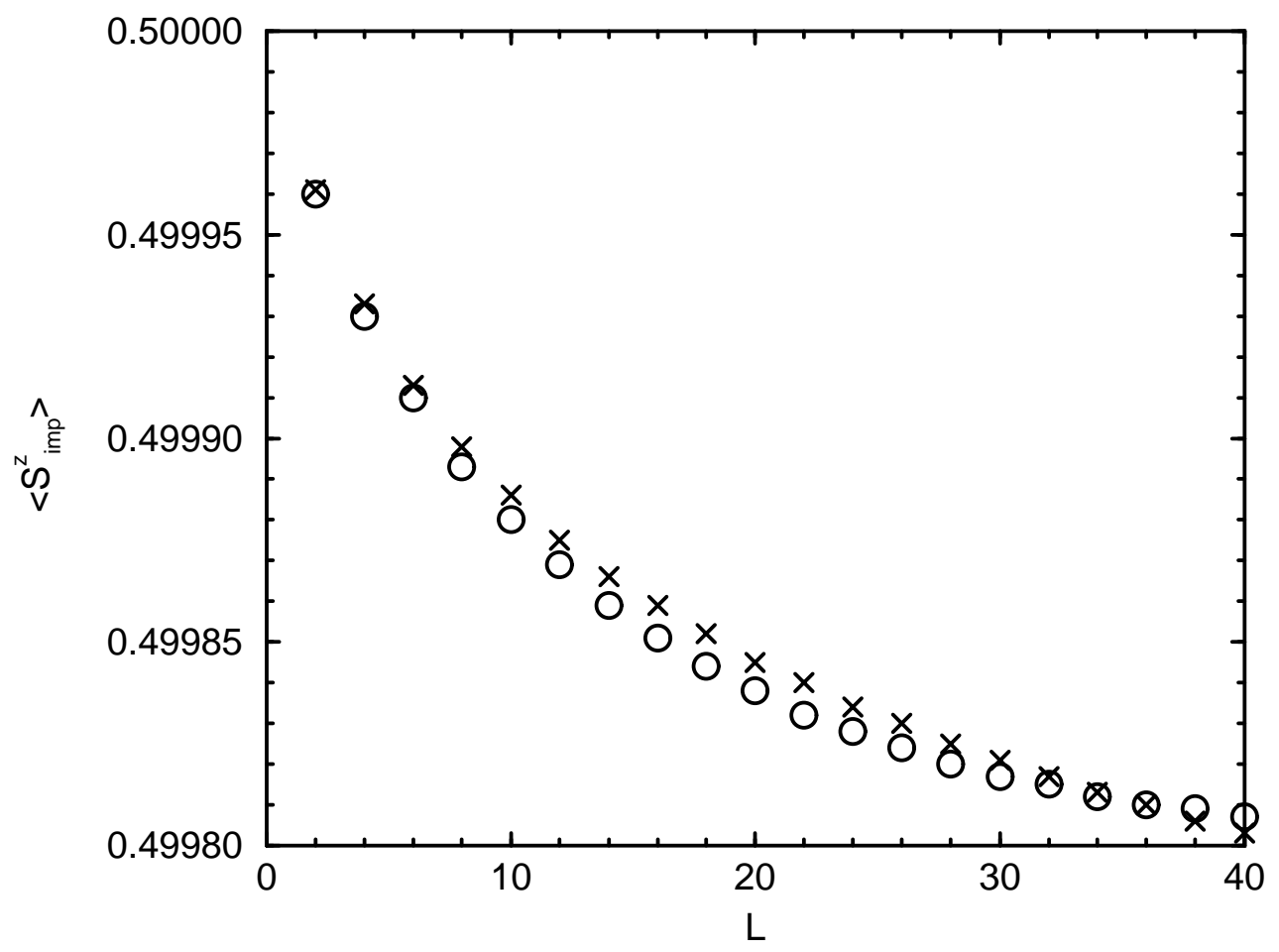

FIG. 3. The z-component of the impurity spin as a function of chain length, $L$. One impurity is present at the left end of the chain. The Kondo coupling is $J=0.05$. The crosses indicate the first order perturbation result, Eq. (6.8), the circles denote DMRG results for the state $S_{T}^{z}=1 / 2, R P H=1$. 


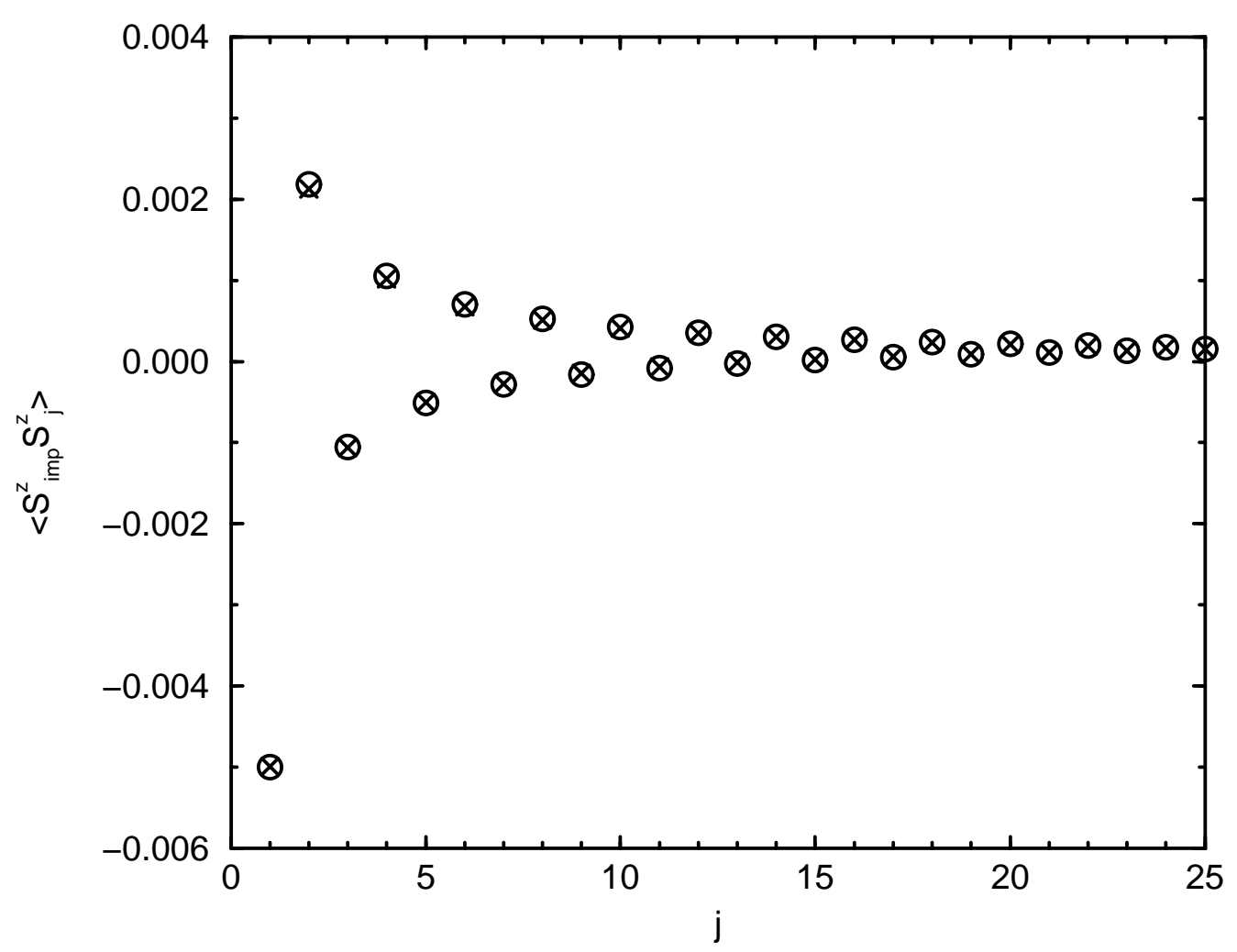

FIG. 4. The correlation function $<S_{\mathrm{imp}}^{z} S_{j}^{z}>$ as a function of site index j (circles), for a chain of length $L=50$. At both ends of the chain are $S=1 / 2$ impurity spins. Only half the chain is shown. The Kondo coupling is $J=0.050, m=150$ states are kept with $R P H=1, P=1$, and $S_{T}^{z}=1$. The circles denote the DMRG results. The crosses indicate the perturbation result, $1 / 2$ times Eq. (6.5). 


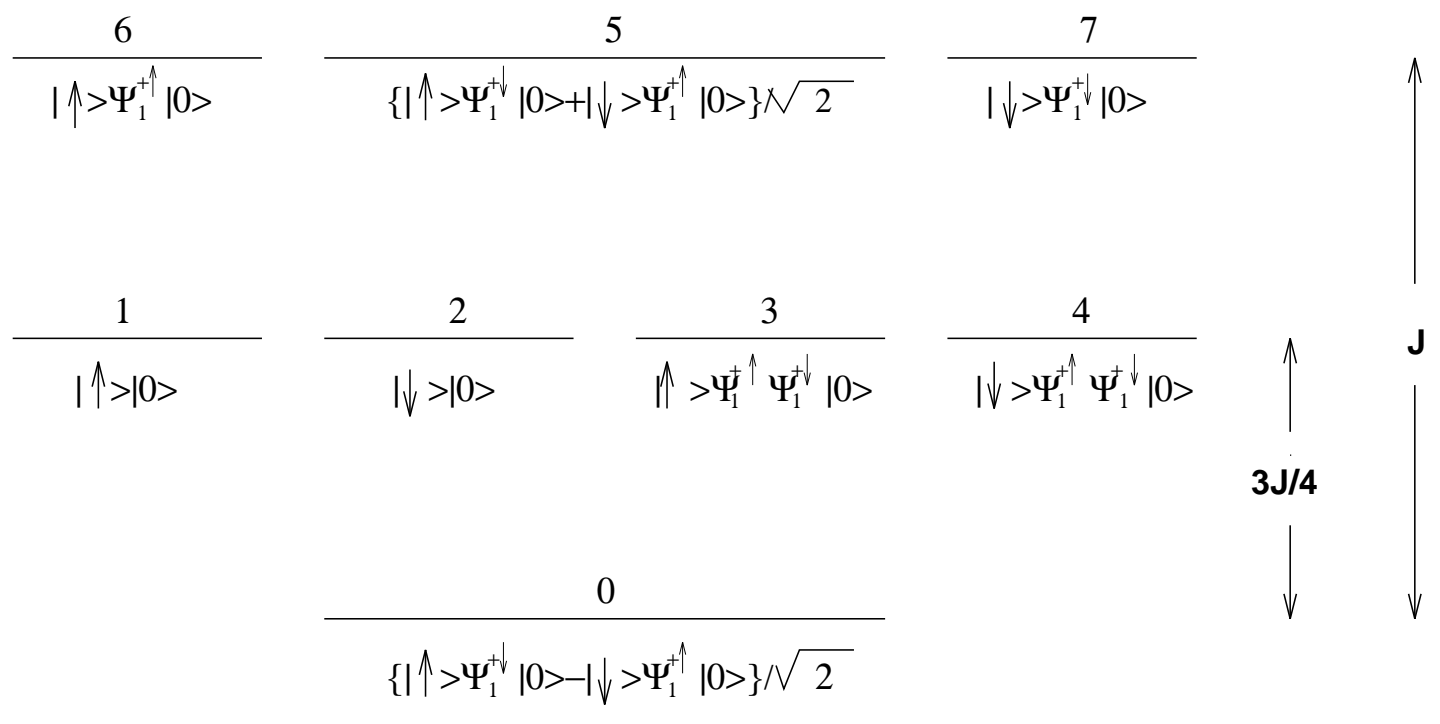

FIG. 5. The 8 states for the impurity site. 


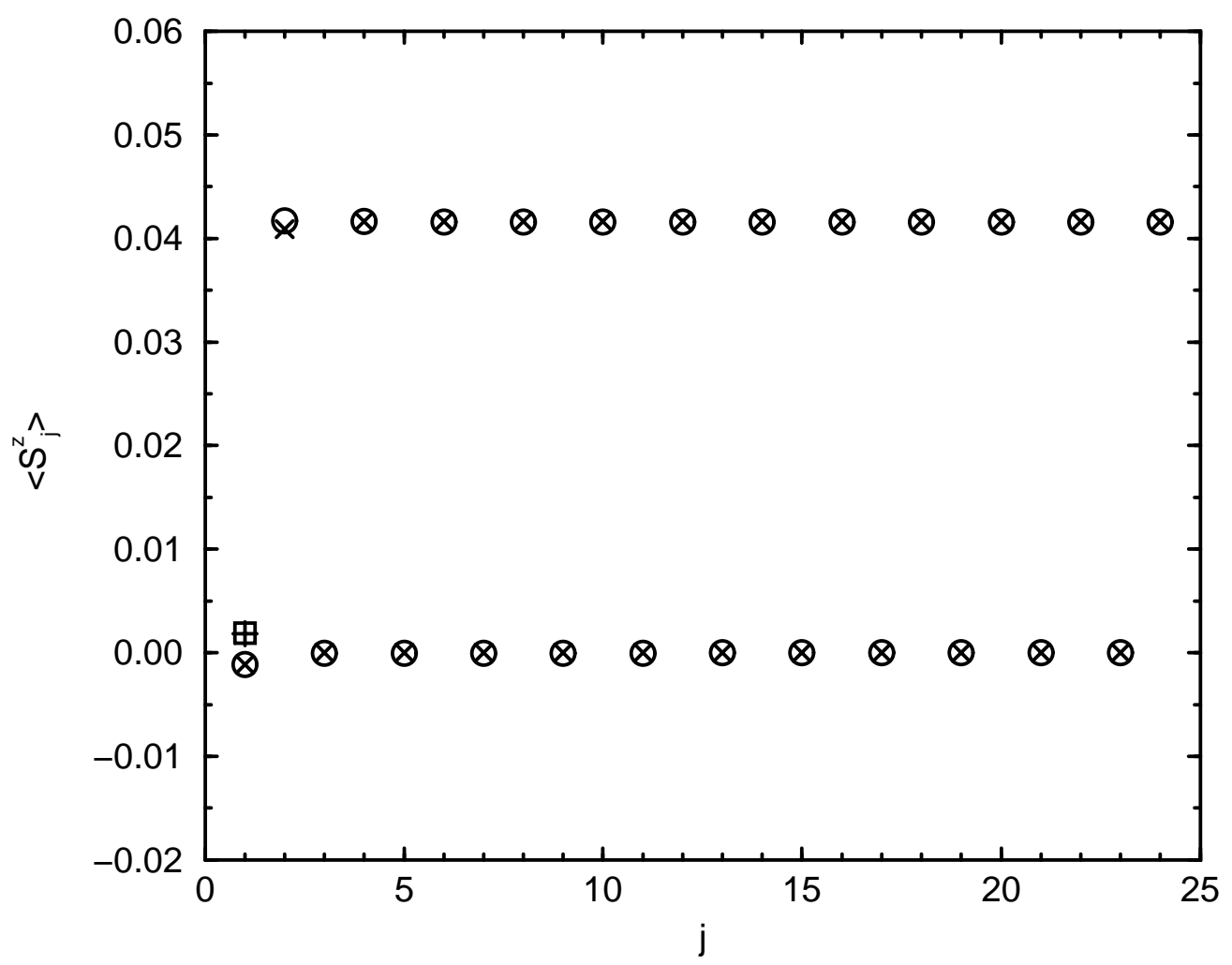

FIG. 6. The z-component of the electron spin as a function of site index $\mathrm{j}$ (circles), for a chain of length $L=24$. At the left end of the chain is a $S=1 / 2$ impurity spin. The Kondo coupling is $J=10, m=128$ states are kept with $R P H=1, S_{T}^{z}=1 / 2$. The square denote the DMRG result for the impurity spin. The crosses indicate the perturbation result, Eqs. (5.6), (7.11) and (7.16). The plus is the result for $<S_{\mathrm{imp}}>$ from Eq. (7.14). 


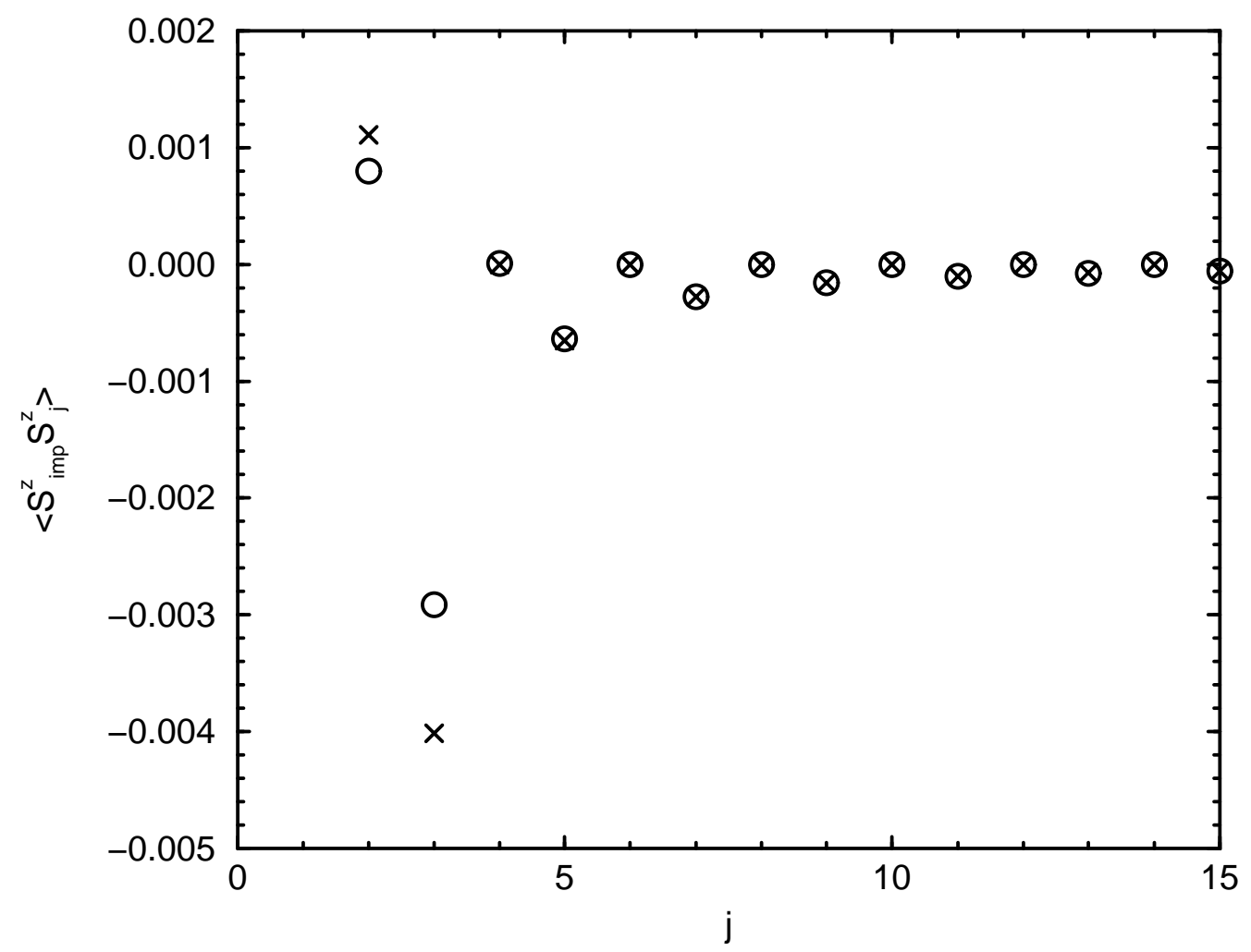

FIG. 7. The correlation function $<S_{\mathrm{imp}}^{z} S_{j}^{z}>$ as a function of site index j (circles), for a chain of length $L=30$. At both ends of the chain are $S=1 / 2$ impurity spins. Only half the chain is shown. The Kondo coupling is $J=10, m=150$ states are kept with $R P H=1, P=-1$, and $S_{T}^{z}=0$. The circles denote the DMRG results. The crosses indicate the perturbation result, Eqs. (7.19) and (7.20). 


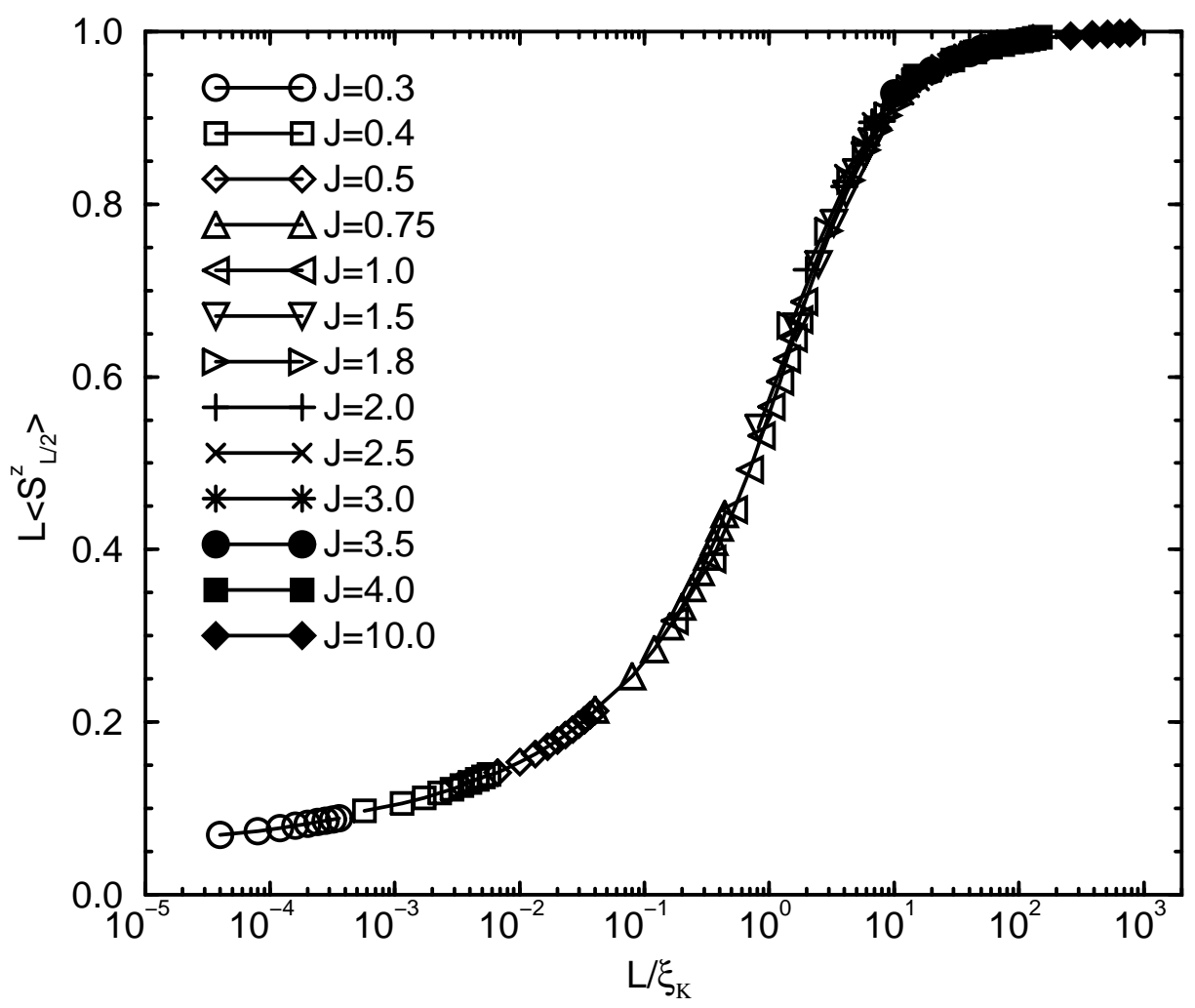

FIG. 8. Logaritmic plot of $L<S_{L / 2}^{z}>$ as a function of chain length $L / \xi_{K}$ for a range of different coupling constants. The initial point corresponds in all cases to $L=4$. The solid lines are guides to the eye. The strong coupling limit corresponds to $L<S_{L / 2}^{z}>\approx 1$. 


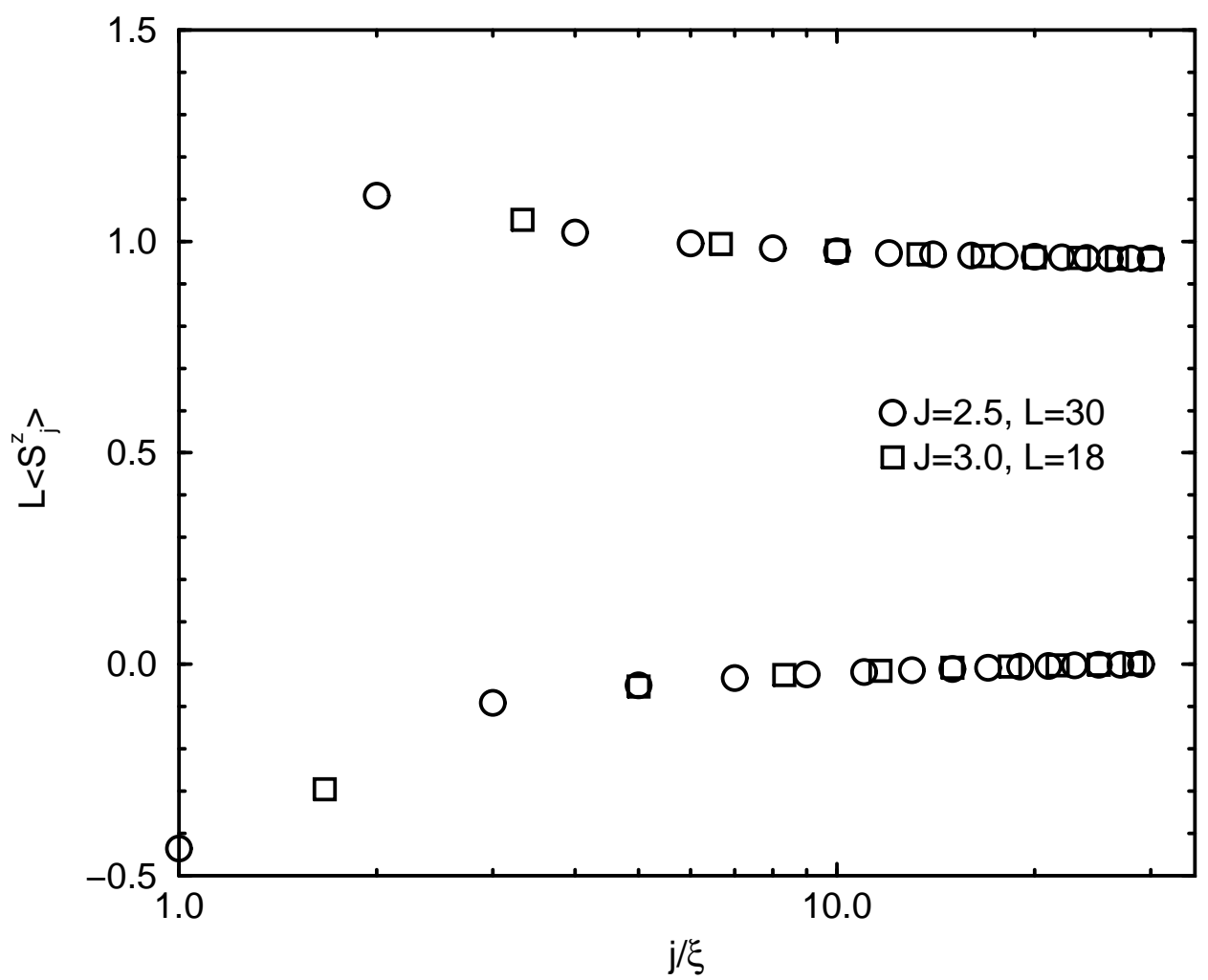

FIG. 9. $L$ times the expectation value of the z-component of the electron spin, $\left\langle S_{j}^{z}>\right.$, as a function of $j / \xi_{K}(J)$. Two systems are shown: $J=2.5, \quad \xi_{K}=1.0, L=30$ and $J=3.0, \xi_{K}=0.6, L=18$ Thus in both cases we have $L / \xi_{K} \approx 30$. Clearly the data collapses onto a universal curve. 


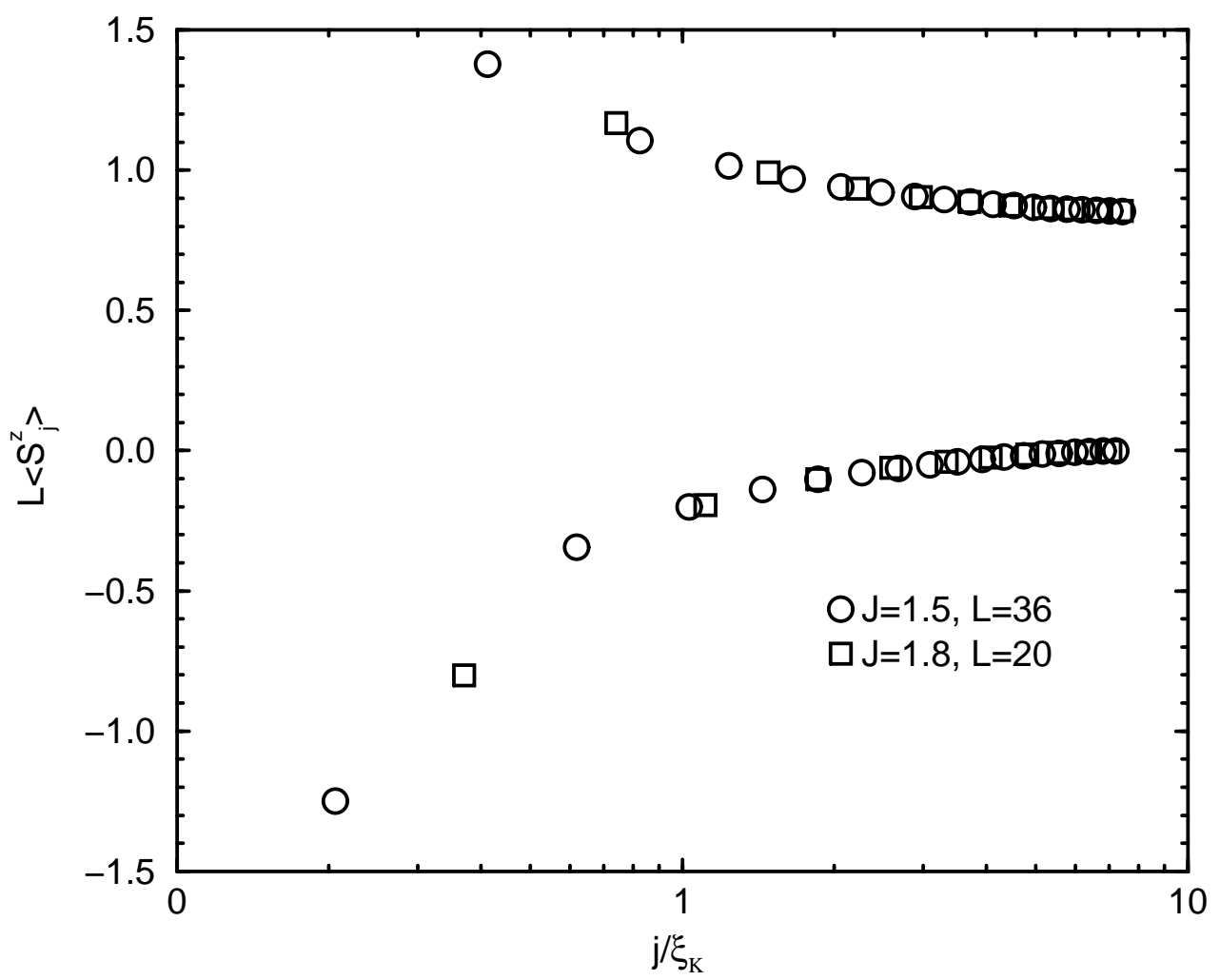

FIG. 10. $L$ times the expectation value of the z-component of the electron spin, $\left\langle S_{j}^{z}\right\rangle$, as a function of $j / \xi_{K}(J)$. Two systems are shown: $J=1.8, \quad \xi_{K}=2.7, L=20$ and $J=1.5, \xi_{K}=4.85, L=36$ Thus in both cases we have $L / \xi_{K} \approx 7.4$. Clearly the data collapses onto a universal curve. 


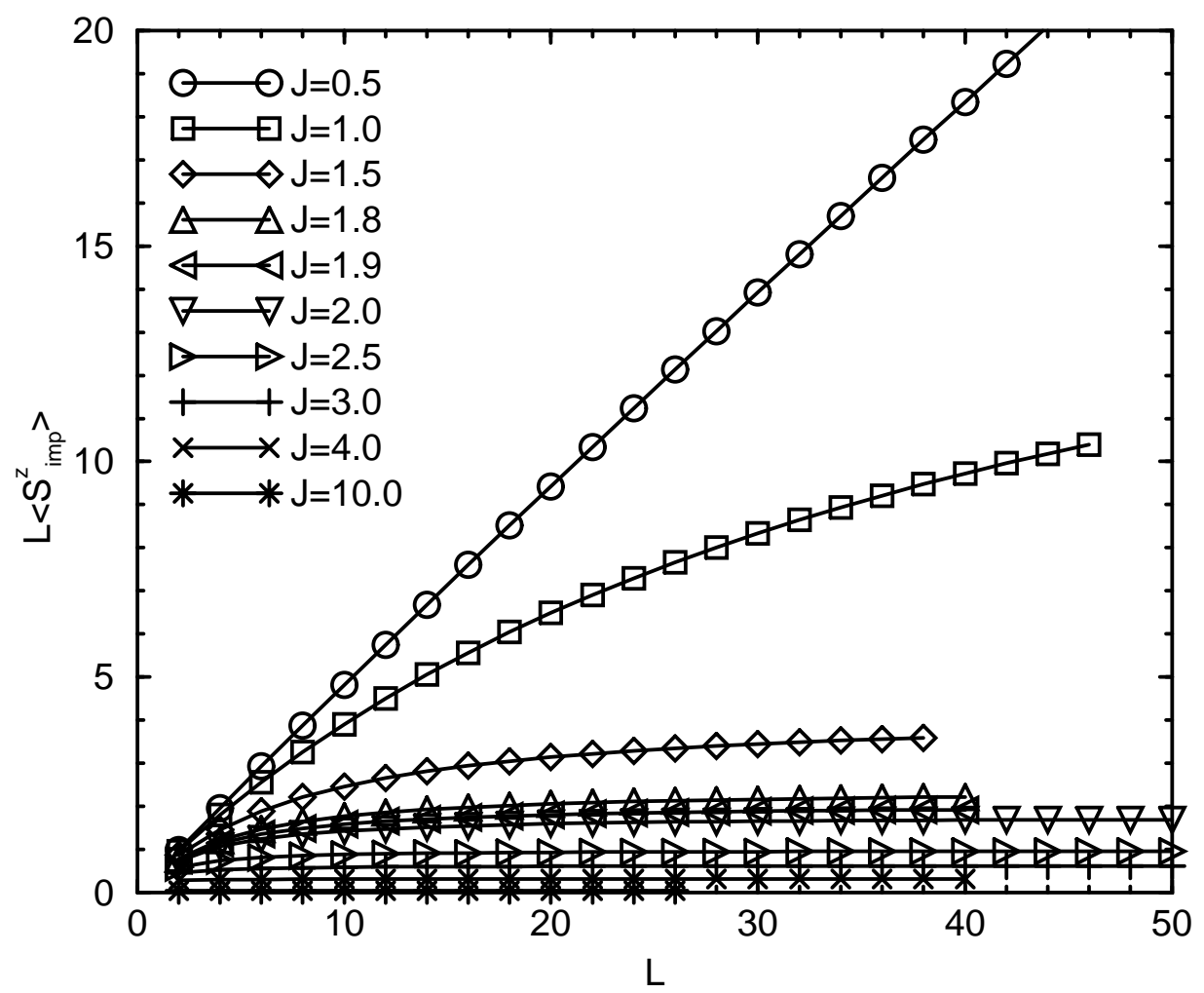

FIG. 11. The scaled z-component of the impurity spin, $L<S_{\mathrm{imp}}^{z}>$, as a function of chain length, $L$, for a range of Kondo couplings $J$. In all cases does the first point correspond to $L=2$. 


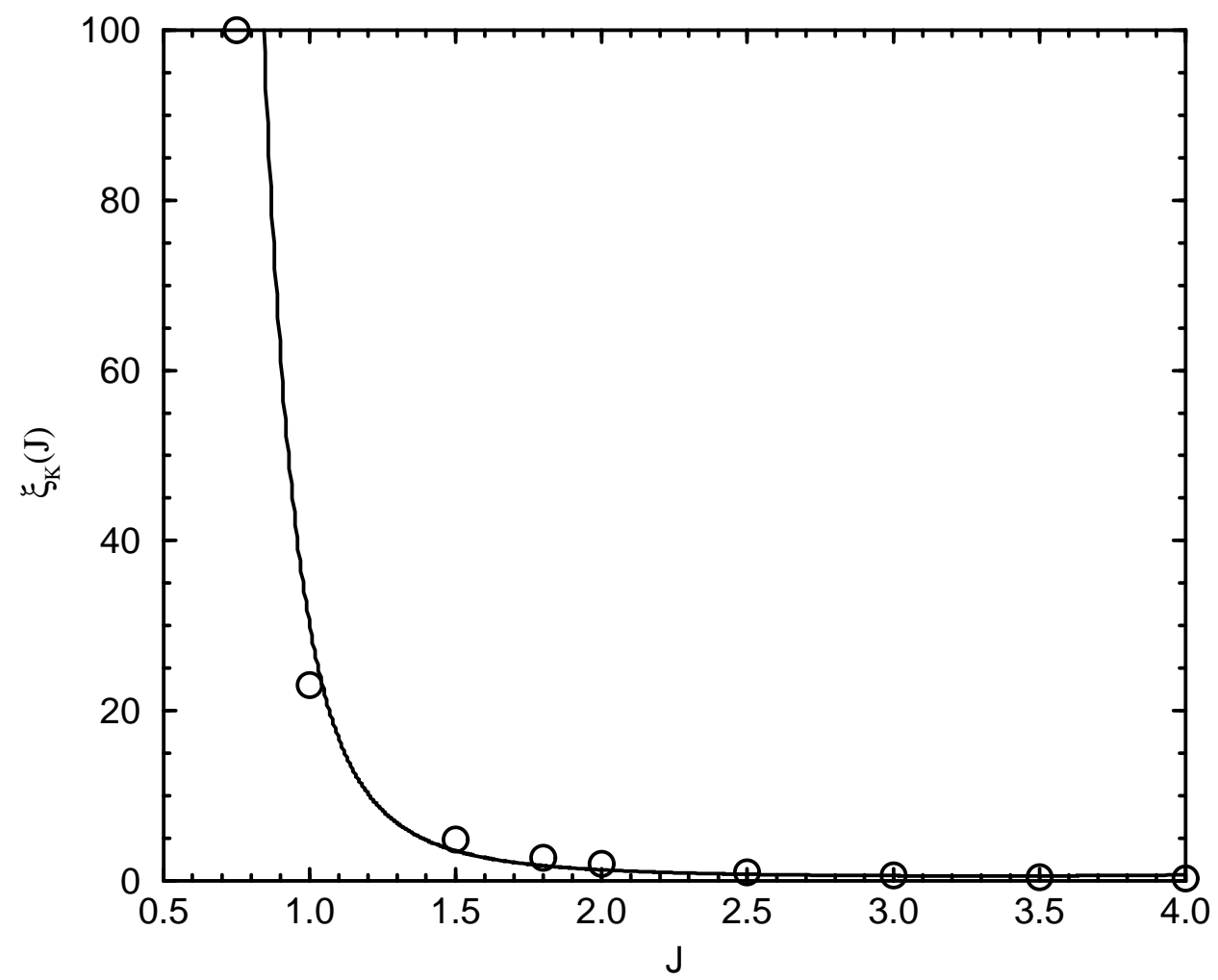

FIG. 12. The Kondo length, $\xi_{K}$, as a function of Kondo coupling, $J$. The circles denote the numerical DMRG results. The solid line indicates a least square fit of the results shown to the form Eq. (8.8). The fitted parameters are $\xi_{0}=1.76, c=0.21$. 